\title{
An enhanced beam-theory model of the mixed-mode bending (MMB) test - Part II: applications and results
}

\author{
Stefano Bennati $\cdot$ Paolo Fisicaro $\cdot$ Paolo S. Valvo \\ Dipartimento di Ingegneria Civile e Industriale, Università di Pisa, Largo Lucio \\ Lazzarino, 56126 Pisa, Italy \\ Phone +390502218200 \\ Fax +390502218201 \\ E-mail p.valvo@ing.unipi.it
}

Abstract The paper presents an enhanced beam-theory (EBT) model of the mixed-mode bending (MMB) test, whereby the specimen is considered as an assemblage of two sublaminates partly connected by an elastic-brittle interface. Analytical expressions for the compliance, energy release rate, and mode mixity are deduced. A compliance calibration strategy enabling numerical or experimental evaluation of the interface elastic constants is also presented. Furthermore, analytical expressions for the crack length correction parameters - analogous to those given by the corrected beam-theory (CBT) model for unidirectional laminated specimens - are furnished for multidirectional laminated specimens, as well. Lastly, an example application to experimental data reduction is presented.

Keywords Mixed-mode bending $(M M B)$ test $\cdot$ Beam-theory model $\cdot$ Finite element analysis $\cdot$ Compliance $\cdot$ Energy release rate $\cdot$ Mode mixity

\section{Introduction}

Since 2001 the mixed-mode bending (MMB) test - introduced by Reeder and Crews in 1988 [1, 2] and later refined [3, 4] - has been adopted by ASTM as the standard test method for I/II mixed-mode interlaminar fracture toughness of unidirectional fibre-reinforced composite laminates [5]. The MMB test can be regarded as the superposition of pure mode I and mode II tests, namely, the double cantilever beam (DCB) and end-notched flexure (ENF) tests, respectively [6].

Several mechanical models of the MMB test have been proposed in the literature. Here we limit ourselves to recalling the main analytical models, whereas Part I [7] of this paper contains a more detailed review of the literature on the subject. 
In the simple beam-theory (SBT) model, the specimen is considered as an assemblage of three rigidly connected sublaminates, each of which is modelled as an Euler-Bernoulli beam [2], while the Timoshenko beam-theory (TBT) model accounts for the transverse shear deformability of the sublaminates as well. Kinloch et al. [8] have proposed a corrected beam-theory (CBT) model, whereby the effects of deflections and rotations at the crack tip are taken into account by considering increased delamination lengths. These are defined through crack length correction parameters, for which several expressions have been suggested in the literature. Williams [9] has obtained an analytical expression for the mode I correction parameter by starting with Kanninen's solution for a DCB test specimen [10] and introducing some semi-empirical corrections to better match the results of finite element analyses. Subsequently, Wang and Williams [11] suggested calculating the mode II correction parameter simply as a fraction of the corresponding mode I correction parameter. The resulting definitions of the mode I and II crack length correction parameters yield good results for both isotropic and orthotropic homogeneous specimens as well as for unidirectional (UD) laminated specimens [12]. The current ASTM standard thus suggests using the CBT model for experimental data reduction when characterising UD laminates [5].

Subsequent studies have been devoted to more accurate estimation of the mode II crack length correction parameter. For orthotropic specimens, analytical expressions have been given by Wang and Qiao [13], de Morais [14], and Jumel et al. [15].

Developing a mechanical model of the MMB test for cases of multidirectional (MD) and asymmetric laminated specimens is thus still an open issue. For MD laminated specimens, Pereira and de Morais have proposed a modified beamtheory (MBT) model, whereby an additional term accounts for the transverse shear deformability in the mode II compliance and the crack length correction parameters are computed by considering the homogenised flexural and shear moduli [16-18]. More recently, in an effort to model MD asymmetric specimens, the same authors have suggested using different crack length correction parameters for the upper and lower sublaminates [19].

Following a modelling approach already adopted for the asymmetric double cantilever beam (ADCB) test [20], we have formulated an enhanced beam-theory 
(EBT) model of the MMB test. Accordingly, the laminated specimen is considered as an assemblage of two sublaminates - modelled as extensible, flexible, and shear-deformable beams - partly connected by an elastic-brittle interface. The EBT model can be applied to both UD and MD laminated specimens, as well as to adhesively bonded specimens, provided that the following general hypotheses are fulfilled:

a) the delamination splits the specimen into two sublaminates having identical mechanical properties;

b) the sublaminates behave as plane beams and exhibit neither shear-extension nor bending-extension coupling;

c) non-linear effects are negligible.

In Part I of this paper [7], the problem has been formulated using a set of differential equations for which a complete explicit solution has been deduced, including analytical expressions for the internal forces, interfacial stresses, and displacements. The solution obtained will now be applied to determine analytical expressions for an MMB test specimen's compliance, energy release rate, and mode mixity. Furthermore, analytical expressions for the abovementioned quantities will also be given for the DCB and ENF test specimens, to which the proposed general solution applies as special cases. Comparisons are presented between the analytical predictions of the enhanced beam-theory model and simpler analytical models reported in the literature. Moreover, the predictions of the proposed model are compared with the results of numerical analyses performed via an expressly developed finite element model. The finite element model is also used to evaluate the elastic constants of the interface of the EBT model through a numerical compliance calibration strategy. Lastly, we present an application of the model to cases of UD and MD laminated specimens for which experimental and numerical results have been drawn from the literature.

\section{The EBT model of the MMB test}

We consider an MMB test specimen [1-5] of length $L$, width $B$ and thickness $H=$ $2 h$, affected at one of its ends by a delamination of length $a$, which splits the laminate into two sublaminates having identical mechanical properties (Fig. 1).

In the EBT model [7] the sublaminates may have any stacking sequence, provided that they behave as plane beams and exhibit neither shear-extension nor 
bending-extension coupling [21]. This condition is fulfilled not only by homogenous and unidirectional laminated specimens, but also by symmetric cross-ply and angle-ply specimens, as well as more generic uncoupled multidirectional laminated specimens [22]. Global reference $x$ - and $z$-axes are fixed, aligned with the specimen's axial and transverse directions, respectively. An abscissa $s$ measures the distances of the sublaminates' cross sections from the crack tip. The specimen is simply supported and subjected to an upward load, $P_{\mathrm{u}}$, and a downward load, $P_{\mathrm{d}}$. We denote with $d$ the distance between $P_{\mathrm{u}}$ and $P_{\mathrm{d}}$ and also define $b=L-a$ and $\ell=L-d$. In conformity with the ASTM standard [5], the downward load, $P_{\mathrm{d}}$, is applied at the specimen's mid-span section, so that $\ell=d=L / 2$.

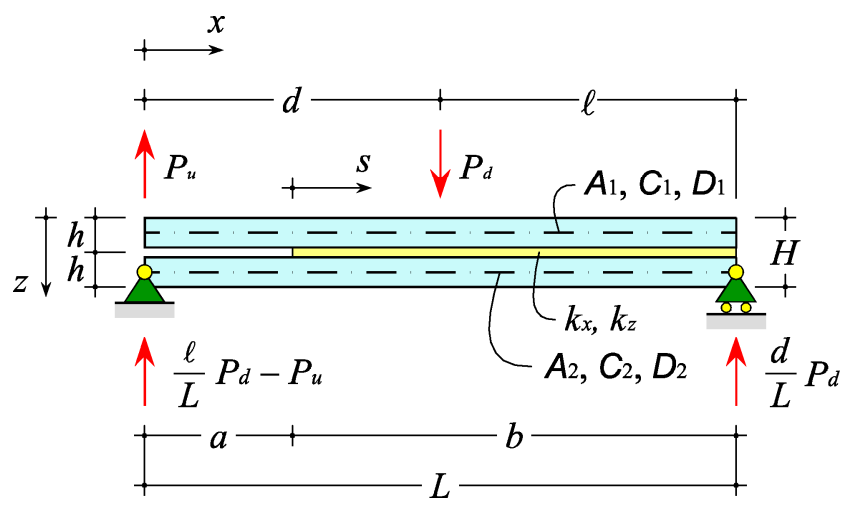

Fig. 1 Enhanced beam-theory model of the MMB test

In line with classical laminated plate theory [21], we denote with $A_{1}=A_{2}$, $C_{1}=C_{2}$, and $D_{1}=D_{2}$ the sublaminates' extensional stiffness, shear stiffness, and bending stiffness, respectively. For homogeneous orthotropic specimens, by denoting $E_{x}, E_{y}, E_{z}$ and $G_{x y}, G_{y z}, G_{z x}$ as the elastic moduli in the fixed reference system, the sublaminates' stiffnesses are $A_{1}=E_{x} h, C_{1}=5 G_{z x} h / 6$, and $D_{1}=E_{x} h^{3} / 12$.

The sublaminates are partly connected by a deformable interface, which is regarded as a continuous distribution of linearly elastic-brittle springs acting along the normal and tangential directions with respect to the interface plane. Correspondingly, we denote with $k_{z}$ and $k_{x}$ the elastic constants of the distributed springs. 


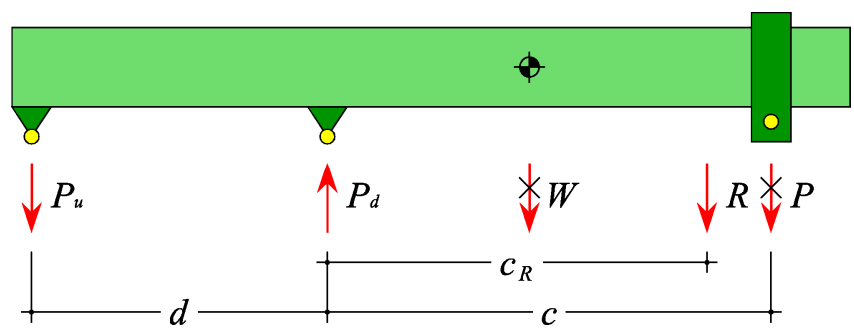

Fig. 2 Loading lever

\section{Compliance}

\subsection{Definition}

Assuming a linearly elastic load-deflection response, the compliance of a test specimen is conventionally defined as $C=\delta / P$, where $P$ is the load applied to the specimen and $\delta$ is the displacement of the load application point [6]. In the MMB test, the load $P$ is applied by the testing machine to a loading lever of weight $W$ (Fig. 2), which in turn transfers the loads $P_{u}$ and $P_{d}$ to the specimen. The intensities of $P_{u}$ and $P_{d}$ are controlled by suitably adjusting the lengths of the lever arms, $c$ and $d$. Accordingly, the compliance is

$C=\frac{\delta_{R}}{R}$,

where $R=P+W$ is the resultant force, and $\delta_{R}$ is the displacement of its application point. Under the usual assumption that the lever behaves as a rigid body, we have

$\delta_{R}=\frac{c_{R}}{d} \delta_{u}+\left(1+\frac{c_{R}}{d}\right) \delta_{d}$

where $\delta_{u}$ and $\delta_{d}$ are the displacements of the application points of $P_{u}$ and $P_{d}$, respectively, and $c_{R}$ is distance between the application points of $P_{d}$ and $R$.

The lever weight $W$ is generally considered negligible with respect to $P$, so we may put $R \cong P, c_{R} \cong c$, and $\delta_{R} \cong \delta$ (according to ASTM standard [5], $W$ would have to be less than $3 \%$ of $P$ to be negligible). In this case, the loads applied to the specimen are

$P_{u}=\frac{c}{d} P \quad$ and $\quad P_{d}=\left(1+\frac{c}{d}\right) P$

and the compliance becomes 
$C \cong \frac{\delta}{P}=\left(\frac{c}{d}\right)^{2} \frac{\delta_{u}}{P_{u}}+\left(1+\frac{c}{d}\right)^{2} \frac{\delta_{d}}{P_{d}}$.
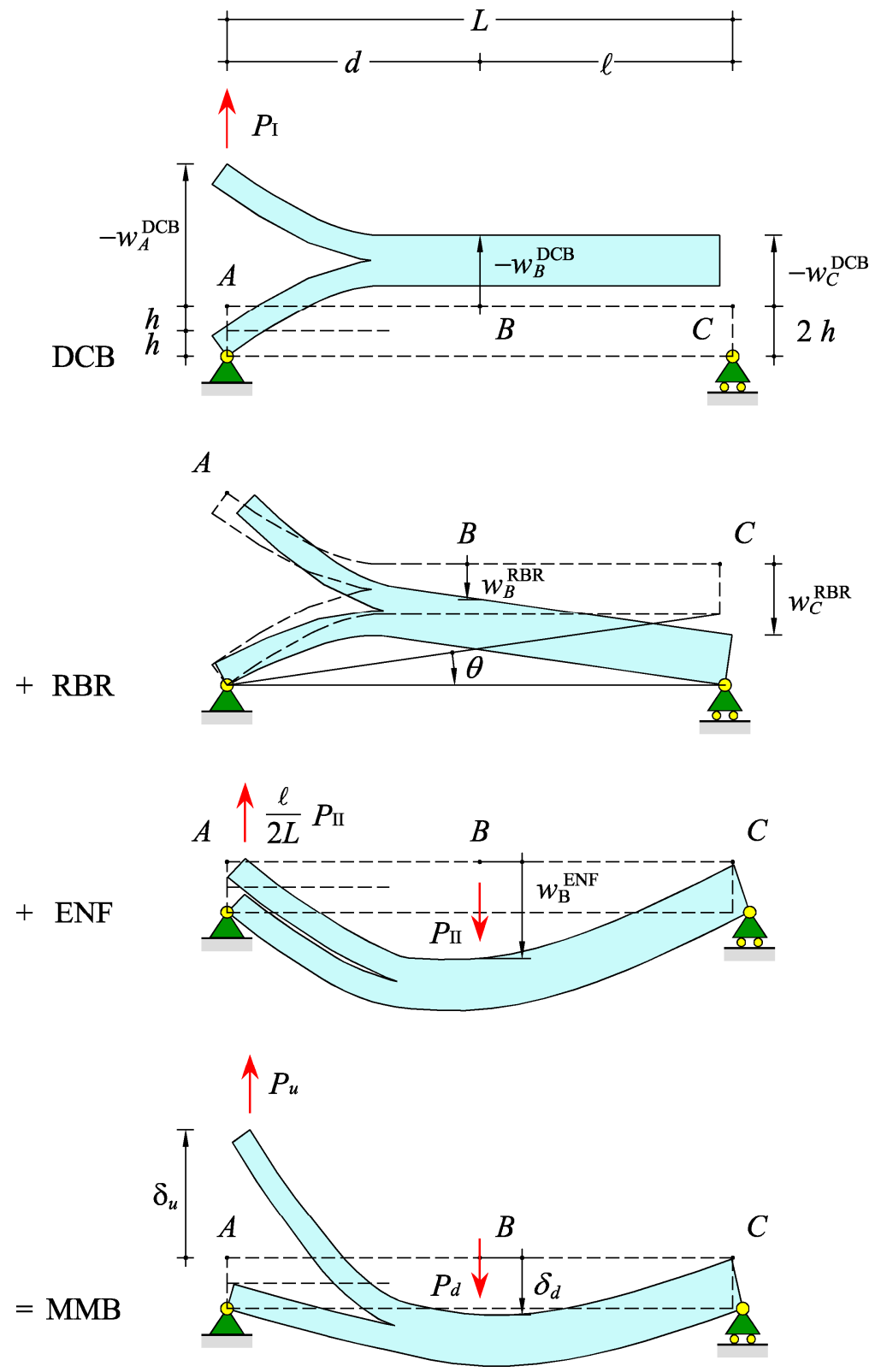

Fig. 3 Displacement superposition for the MMB test

As discussed in Part I [7], from the static point of view the MMB test can be regarded as the superposition of the double cantilever beam (DCB) and endnotched flexure (ENF) tests (respectively corresponding to mode I and II fracture). As far as kinematics is concerned, however, one should bear in mind that different constraints are used in the DCB and ENF tests. Thus, in order to add the displacements of the DCB test to those of the ENF test and obtain the correct displacements of the MMB test, it is necessary to also consider an infinitesimal 
rigid-body rotation (RBR) of an angle $\theta=w_{\mathrm{C}}^{\mathrm{RBR}} / L$ around the specimen's lefthand end hinge (Fig. 3).

Consequently, by denoting with $A$ and $B$ the application points of $P_{u}$ and $P_{d}$, respectively, the corresponding displacements are

$\delta_{u}=-w_{A}^{\mathrm{DCB}} \quad$ and $\quad \delta_{d}=w_{B}^{\mathrm{DCB}}+w_{B}^{\mathrm{RBR}}+w_{B}^{\mathrm{ENF}}$,

where $w$ denotes the transverse displacement (positive if downwards). The specific contributions to Eqs. (5) can be evaluated as follows. Denoting with $C_{\mathrm{DCB}}$ and $C_{\mathrm{ENF}}$ the compliances of the DCB and ENF test specimens, respectively, we can write

$w_{A}^{\mathrm{DCB}}=-P_{\mathrm{I}} C_{\mathrm{DCB}} \quad$ and $\quad w_{B}^{\mathrm{ENF}}=P_{\mathrm{II}} C_{\mathrm{ENF}}$,

where, recalling Eqs. (13) in Part I [7],

$P_{\mathrm{I}}=\frac{1}{2}\left(\frac{c+d}{L}+\frac{c}{d}-1\right) P \quad$ and $\quad P_{\mathrm{II}}=\left(1+\frac{c}{d}\right) P$

are the loads responsible for fracture modes I and II, respectively. Furthermore, supposing that the deformation of the specimen in the thickness direction is negligible, we have

$w_{B}^{\mathrm{DCB}} \cong \frac{w_{A}^{\mathrm{DCB}}}{2}=-\frac{\delta_{u}}{2} \quad$ and $\quad w_{B}^{\mathrm{RBR}}=\theta d \cong-\frac{w_{C}^{\mathrm{DCB}}}{L} d \cong \frac{\delta_{u}}{2} \frac{d}{L}$.

Hence, by substituting Eqs. (6) and (8) into (5), we obtain

$\delta_{u}=P_{\mathrm{I}} C_{\mathrm{DCB}} \quad$ and $\quad \delta_{d}=-\frac{\ell}{2 L} P_{\mathrm{I}} C_{\mathrm{DCB}}+P_{\mathrm{II}} C_{\mathrm{ENF}}$

and the compliance of the MMB test specimen becomes

$C_{\mathrm{MMB}}=\left(\frac{P_{\mathrm{I}}}{P}\right)^{2} C_{\mathrm{DCB}}+\left(\frac{P_{\mathrm{II}}}{P}\right)^{2} C_{\mathrm{ENF}}$.

By substituting equations (7) into (10), we obtain

$C_{\mathrm{MMB}}=\frac{1}{4}\left(\frac{c+d}{L}+\frac{c}{d}-1\right)^{2} C_{\mathrm{DCB}}+\left(1+\frac{c}{d}\right)^{2} C_{\mathrm{ENF}}$

and for a standard MMB test (where $\ell=d=L / 2$ ) [23]

$C_{\mathrm{MMB}}=\left(\frac{3 c-\ell}{4 \ell}\right)^{2} C_{\mathrm{DCB}}+\left(\frac{c+\ell}{\ell}\right)^{2} C_{\mathrm{ENF}}$. 
Eqs. (4), (10), (11) and (12) represent alternative ways to compute the compliance of the MMB test specimen according to any analytical or numerical model, provided that suitable expressions or values of $\delta_{\mathrm{u}}$ and $\delta_{\mathrm{d}}$, or $C_{\mathrm{DCB}}$ and $C_{\mathrm{ENF}}$, are used. However, it should be observed that Eqs. (10)-(12) strictly hold only as long as the approximations behind Eqs. (8) are acceptable.

\subsection{Beam-theory models}

In the simple beam-theory (SBT) and Timoshenko beam-theory (TBT) models [2, 3] the specimen is considered as an assemblage of three rigidly connected sublaminates (Fig. 4). The extensional stiffness, shear stiffness, and bending stiffness of the unbroken part of the specimen are $A_{3}=2 A_{1}, C_{3}=2 C_{1}$, and $D_{3}=2 D_{1}+A_{1} h^{2} / 2$, respectively.

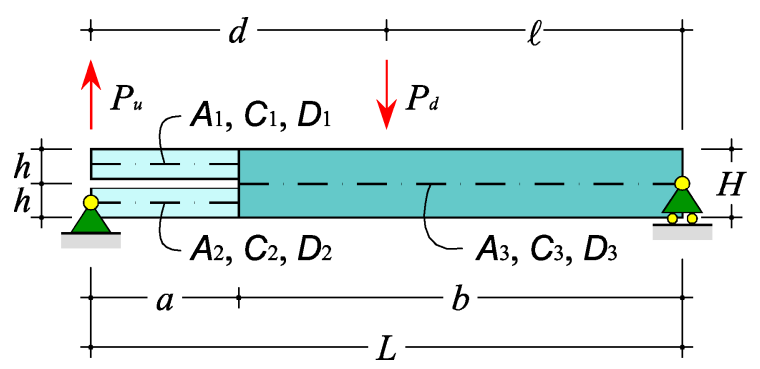

Fig. 4 SBT and TBT models of the MMB test

In the SBT model the sublaminates are modelled as Euler-Bernoulli beams, so that $C_{1}, C_{2}, C_{3} \rightarrow \infty$. Thus, considering a standard MMB test (where $\ell=d=L / 2$ ), the compliances of the DCB and ENF test specimens are respectively

$C_{\mathrm{DCB}}^{\mathrm{SBT}}=\frac{2 a^{3}}{3 B D_{1}} \quad$ and $\quad C_{\mathrm{ENF}}^{\mathrm{SBT}}=\frac{1}{24 B} \frac{A_{1} h^{2}}{A_{1} h^{2}+4 D_{1}}\left(\frac{a^{3}}{D_{1}}+\frac{8 \ell^{3}}{A_{1} h^{2}}\right)$

Thus, from equation (12), the compliance of the MMB test specimen is

$C_{\mathrm{MMB}}^{\mathrm{SBT}}=\frac{1}{24 B}\left[\left(\frac{3 c-\ell}{\ell}\right)^{2} \frac{a^{3}}{D_{1}}+\left(\frac{c+\ell}{\ell}\right)^{2} \frac{A_{1} h^{2}}{A_{1} h^{2}+4 D_{1}}\left(\frac{a^{3}}{D_{1}}+\frac{8 \ell^{3}}{A_{1} h^{2}}\right)\right]$,

and in particular for orthotropic specimens we have [5]

$C_{\mathrm{DCB}}^{\mathrm{SBT}}=\frac{8 a^{3}}{B E_{x} h^{3}} \quad$ and $\quad C_{\mathrm{ENF}}^{\mathrm{SBT}}=\frac{3 a^{3}+2 \ell^{3}}{8 B E_{x} h^{3}}$, 
and

$$
C_{\mathrm{MMB}}^{\mathrm{SBT}}=\frac{1}{2 B E_{x} h^{3}}\left[\left(\frac{3 c-\ell}{\ell}\right)^{2} a^{3}+\frac{1}{4}\left(\frac{c+\ell}{\ell}\right)^{2}\left(3 a^{3}+2 \ell^{3}\right)\right] .
$$

In the TBT model the contributions to the displacements stemming from the sublaminates' shear stiffnesses are considered at the first order [24], hence suitable correction terms are introduced into expressions (13),

$$
C_{\mathrm{DCB}}^{\mathrm{TBT}}=C_{\mathrm{DCB}}^{\mathrm{SBT}}+\frac{2 a}{B C_{1}} \quad \text { and } \quad C_{\mathrm{ENF}}^{\mathrm{TBT}}=C_{\mathrm{ENF}}^{\mathrm{SBT}}+\frac{\ell}{4 B C_{1}},
$$

and from Eq. (12) the specimen's compliance becomes

$$
C_{\mathrm{MMB}}^{\mathrm{TBT}}=C_{\mathrm{MMB}}^{\mathrm{SBT}}+\frac{1}{4 B C_{1}}\left[\frac{1}{2}\left(\frac{3 c-\ell}{\ell}\right)^{2} a+\left(\frac{c+\ell}{\ell}\right)^{2} \ell\right] .
$$

\subsection{Corrected beam-theory model}

Kinloch et al. [8] have proposed a corrected beam-theory (CBT) model, whereby the effects of deflections and rotations at the crack tip - ignored in the SBT and TBT models - are taken into account approximately by considering the increased delamination lengths,

$a_{\mathrm{I}}^{\mathrm{CBT}}=a+\chi_{\mathrm{I}} h \quad$ and $\quad a_{\mathrm{II}}^{\mathrm{CBT}}=a+\chi_{\mathrm{II}} h$,

where $\chi_{\mathrm{I}}$ and $\chi_{\mathrm{II}}$ are the respective correction parameters for fracture modes I and II.

For unidirectional laminated specimens, the ASTM standard [5] suggests:

$$
\chi_{\mathrm{I}}=\sqrt{\frac{E_{x}}{11 G_{z x}}\left[3-2\left(\frac{\Gamma}{1+\Gamma}\right)^{2}\right]} \text { and } \chi_{\mathrm{II}}=0.42 \chi_{\mathrm{I}} \text {, }
$$

where $\Gamma=1.18 \sqrt{E_{x} E_{z}} / G_{z x}$. Accordingly, the compliance is obtained by properly introducing the increased delamination lengths (19) into Eq. (15) [4],

$$
C_{\mathrm{MMB}}^{\mathrm{CBT}}=\frac{1}{2 B E_{x} h^{3}}\left\{\left(\frac{3 c-\ell}{\ell}\right)^{2}\left(a+\chi_{\mathrm{I}} h\right)^{3}+\frac{1}{4}\left(\frac{c+\ell}{\ell}\right)^{2}\left[3\left(a+\chi_{\mathrm{II}} h\right)^{3}+2 \ell^{3}\right]\right\} .
$$

Note that the mode I crack length correction parameter, $\chi_{\mathrm{I}}$, already includes a term for the transverse shear deformability through the elastic shear modulus $G_{z x}$ 
[9], while for the mode II contribution an additional term should be introduced into the expression for $C_{\mathrm{ENF}}[16]$.

For laminates with generic stacking sequences, Eqs. (20) cannot be used. Thus, de Morais and Pereira [18] propose evaluating the correction parameters by considering the homogenised flexural and shear moduli in place of $E_{x}$ and $G_{z x}$, respectively.

\subsection{Enhanced beam-theory model}

In the enhanced beam-theory (EBT) model, the crack-tip deflections and rotations are taken into account by considering a deformable interface connecting the sublaminates. In order to deduce an expression for the compliance, we first determine the expressions for the displacements of the application points of the upward and downward loads, $\delta_{u}$ and $\delta_{d}$. Starting with the analytical solution given in Section 4.4.2 of Part I [7], after some calculations omitted here for brevity, we obtain the upward load displacement

$\delta_{u}^{\mathrm{EBT}}=\delta_{u}^{\mathrm{TBT}}+\delta_{u}^{\mathrm{interface}}$,

where

$\delta_{u}^{\mathrm{TBT}}=\frac{2 P_{\mathrm{I}}}{3 B}\left(\frac{a^{3}}{D_{1}}+\frac{3 a}{C_{1}}\right)$

is the displacement predicted by the Timoshenko beam-theory model and

$\delta_{u}^{\text {interface }}=-\frac{2 P_{\mathrm{I}}}{B D_{1}}\left[\frac{b_{2}}{\lambda_{2}^{2}}+\frac{b_{4}}{\lambda_{1}^{2}}+a\left(\frac{b_{3}}{\lambda_{1}}+\frac{b_{5}}{\lambda_{2}}\right)\right]$

is the additional contribution due to deformation of the elastic interface. In Eq. (24),

$\lambda_{1}=\sqrt{\frac{k_{z}}{C_{1}}\left(1+\sqrt{1-\frac{2 C_{1}^{2}}{k_{z} D_{1}}}\right)}$ and $\lambda_{2}=\sqrt{\frac{k_{z}}{C_{1}}\left(1-\sqrt{1-\frac{2 C_{1}^{2}}{k_{z} D_{1}}}\right)}$

are roots of the characteristic equation of the governing differential problem, and $b_{2}, b_{3}, b_{4}$, and $b_{5}$ are constants characterising the analytical solution, whose exact expressions are given in Part I [7]. However, for the geometry and material properties of typical fibre-reinforced laminated specimens, the following approximate expressions can be used: 


$$
b_{2} \cong-\frac{1+\lambda_{2} a}{\lambda_{1}-\lambda_{2}}, \quad b_{3} \cong \frac{1+\lambda_{2} a}{\lambda_{1}-\lambda_{2}}, \quad b_{4} \cong \frac{1+\lambda_{1} a}{\lambda_{1}-\lambda_{2}}, \quad b_{5} \cong-\frac{1+\lambda_{1} a}{\lambda_{1}-\lambda_{2}} .
$$

Hence, by substituting Eqs. (23), (24) and (26) into (22), we obtain

$$
\delta_{u}^{\mathrm{EBT}} \cong \frac{2 P_{\mathrm{I}} a^{3}}{3 B D_{1}}+\frac{2 P_{\mathrm{I}} a}{B C_{1}}+\frac{2 P_{\mathrm{I}}}{B D_{1}} \frac{1}{\lambda_{1} \lambda_{2}}\left[\left(\lambda_{1}+\lambda_{2}\right) a^{2}+2 a+\frac{1}{\lambda_{1}}+\frac{1}{\lambda_{2}}\right]
$$

Likewise, the downward load displacement turns out to be the sum of four contributions:

$$
\delta_{d}^{\mathrm{EBT}}=\delta_{d, \mathrm{I}}^{\mathrm{TBT}}+\delta_{d, \mathrm{II}}^{\mathrm{TBT}}+\delta_{d, \mathrm{I}}^{\mathrm{interface}}+\delta_{d, \mathrm{II}}^{\mathrm{interface}}
$$

where

$$
\delta_{d, \mathrm{I}}^{\mathrm{TBT}}=-\frac{P_{\mathrm{I}}}{6 B}\left(\frac{a^{3}}{D_{1}}+\frac{3 a}{C_{1}}\right), \quad \text { and } \quad \delta_{d, \mathrm{II}}^{\mathrm{TBT}}=\frac{P_{\mathrm{II}}}{12 B}\left[\frac{1}{2} \frac{A_{1} h^{2}}{A_{1} h^{2}+4 D_{1}}\left(\frac{a^{3}}{D_{1}}+\frac{8 \ell^{3}}{A_{1} h^{2}}\right)+\frac{3 \ell}{C_{1}}\right]
$$

are the contributions predicted by the TBT model, related to the loads responsible for fracture modes I and II, respectively, and

$$
\begin{aligned}
\delta_{d, \mathrm{I}}^{\text {interface }} & =\frac{P_{\mathrm{I}}}{2 B D_{1}}\left[\frac{b_{2}}{\lambda_{2}^{2}}+\frac{b_{4}}{\lambda_{1}^{2}}+a\left(\frac{b_{3}}{\lambda_{1}}+\frac{b_{5}}{\lambda_{2}}\right)\right]-\left.\frac{1}{2} \Delta w\right|_{s=\ell-a}+\left.\frac{1}{4} \Delta w\right|_{s=b}, \\
\delta_{d, \mathrm{II}}^{\text {interface }} & =\frac{P_{\mathrm{II}}}{8 B D_{1}} \frac{A_{1} h^{2}}{A_{1} h^{2}+4 D_{1}} \frac{1}{\lambda_{5}^{2}}\left\{\frac{1}{\tanh \lambda_{5} b} \lambda_{5} a^{2}+a+2 \ell+\right. \\
& \left.-4 \frac{\sinh \lambda_{5} \ell}{\sinh \lambda_{5} b}\left[a+\frac{1}{\lambda_{5}} \sinh \lambda_{5}(b-\ell)\right]\right\}
\end{aligned}
$$

are the additional contributions due to deformation of the elastic interface, again related to $P_{\mathrm{I}}$ and $P_{\mathrm{II}}$, respectively. In Eqs. (30),

$$
\lambda_{5}=\sqrt{2 k_{x}\left(\frac{1}{A_{1}}+\frac{h^{2}}{4 D_{1}}\right)}
$$

is a root of the characteristic equation of the governing differential problem, and $\Delta w$ is the transverse relative displacement at the interface, whose expression is given by Eqs. (54) in Part I [7]. The terms that depend on $\Delta w$, computed at sections far from the crack tip, turn out to be negligible for values corresponding to common composite laminates. Consequently, from comparison of Eqs. (23)(24) and (29)-(30), it follows that

$$
\delta_{d, \mathrm{I}}^{\mathrm{TBT}}=-\frac{1}{4} \delta_{u}^{\mathrm{TBT}} \quad \text { and } \quad \delta_{d, \mathrm{I}}^{\text {interface }} \cong-\frac{1}{4} \delta_{u}^{\text {interface }} .
$$


Therefore, except for the negligible contribution of $\Delta w$, the contributions to $\delta_{d}^{\mathrm{EBT}}$ related to $P_{\mathrm{I}}$ correspond to the infinitesimal rigid-body rotation discussed in Section 3.1.

To sum up, by substituting Eqs. (29), (30) and (32) into (28), and considering the same simplifying assumptions that lead to Eq. (27), we obtain

$$
\begin{aligned}
\delta_{d}^{\mathrm{EBT}} & \cong-\frac{1}{4} P_{\mathrm{I}}\left\{\frac{2 a^{3}}{3 B D_{1}}+\frac{2 a}{B C_{1}}+\frac{2}{B D_{1}} \frac{1}{\lambda_{1} \lambda_{2}}\left[\left(\lambda_{1}+\lambda_{2}\right) a^{2}+2 a+\frac{1}{\lambda_{1}}+\frac{1}{\lambda_{2}}\right]\right\}+ \\
& +P_{\mathrm{II}}\left\{\frac{1}{24 B} \frac{A_{1} h^{2}}{A_{1} h^{2}+4 D_{1}}\left(\frac{a^{3}}{D_{1}}+\frac{8 \ell^{3}}{A_{1} h^{2}}\right)+\frac{\ell}{4 B C_{1}}+\right. \\
& \left.+\frac{1}{8 B D_{1}} \frac{A_{1} h^{2}}{A_{1} h^{2}+4 D_{1}} \frac{1}{\lambda_{5}^{2}}\left[\lambda_{5} a^{2}+a+2 \ell-\frac{2}{\lambda_{5}}-\frac{4 a}{\exp \lambda_{5}(\ell-a)}\right]\right\} .
\end{aligned}
$$

By comparing Eqs. (27) and (33) with Eq. (9), it follows that

$$
\begin{aligned}
& C_{\mathrm{DCB}}^{\mathrm{EBT}} \cong \frac{2 a^{3}}{3 B D_{1}}+\frac{2 a}{B C_{1}}+\frac{2}{B D_{1}} \frac{1}{\lambda_{1} \lambda_{2}}\left[\left(\lambda_{1}+\lambda_{2}\right) a^{2}+2 a+\frac{1}{\lambda_{1}}+\frac{1}{\lambda_{2}}\right], \\
& C_{\mathrm{ENF}}^{\mathrm{EBT}} \cong \frac{1}{24 B} \frac{A_{1} h^{2}}{A_{1} h^{2}+4 D_{1}}\left(\frac{a^{3}}{D_{1}}+\frac{8 \ell^{3}}{A_{1} h^{2}}\right)+\frac{\ell}{4 B C_{1}}+ \\
&+\frac{1}{8 B D_{1}} \frac{A_{1} h^{2}}{A_{1} h^{2}+4 D_{1}} \frac{1}{\lambda_{5}^{2}}\left[\lambda_{5} a^{2}+a+2 \ell-\frac{2}{\lambda_{5}}-\frac{4 a}{\exp \lambda_{5}(\ell-a)}\right]
\end{aligned}
$$

are the compliances predicted by the EBT model for the DCB and ENF test specimens, which can be considered as special cases of our general solution corresponding to $P_{\mathrm{II}}=0$ and $P_{\mathrm{I}}=0$, respectively.

In particular, for orthotropic specimens, the expressions for the compliances of the DCB and ENF test specimens become

$$
\begin{aligned}
& C_{\mathrm{DCB}}^{\mathrm{EBT}} \cong \frac{8 a^{3}}{B E_{x} h^{3}}+\frac{12 a}{5 B G_{z x} h}+\frac{24}{B E_{x} h^{3}} \frac{1}{\lambda_{1} \lambda_{2}}\left[\left(\lambda_{1}+\lambda_{2}\right) a^{2}+2 a+\frac{1}{\lambda_{1}}+\frac{1}{\lambda_{2}}\right], \\
& C_{\mathrm{ENF}}^{\mathrm{EBT}} \cong \frac{3 a^{3}+2 \ell^{3}}{8 B E_{x} h^{3}}+\frac{3 \ell}{10 B G_{z x} h}+\frac{9}{8 B E_{x} h^{3}} \frac{1}{\lambda_{5}^{2}}\left[\lambda_{5} a^{2}+a+2 \ell-\frac{2}{\lambda_{5}}-\frac{4 a}{\exp \lambda_{5}(\ell-a)}\right] .
\end{aligned}
$$

The MMB specimen's compliance can then be obtained by substituting Eqs. (34) or (35) into (12). It is worth noting that the expression for $C_{\mathrm{DCB}}^{\mathrm{EBT}}$ depends on $k_{z}$ (through $\lambda_{1}$ and $\lambda_{2}$ ) and not on $k_{x}$ and, conversely, the expression for $C_{\mathrm{ENF}}^{\mathrm{EBT}}$ depends on $k_{x}$ (through $\lambda_{5}$ ) and not on $k_{z}$. Such observations prepare the ground 
for applying the compliance calibration strategy, illustrated in the following Section.

\subsection{Finite element model and compliance calibration strategy}

In order to have an additional basis for comparison of the analytical models presented in previous Sections, we have built a plane-stress finite element model of a test specimen (Fig. 5a), using the 8-node isoparametric quadrilateral elements QUAD8 available in the Strand7 software [25]. The material has been defined as linearly elastic and orthotropic. Suitable nodal loads and translational restraints have been introduced in order to model the DCB, ENF, and MMB test conditions. Linear static analysis has been carried out. The same geometrical and mechanical properties of the numerical example presented in Part I [7] have been considered. The specimen has span $L=2 \ell=100 \mathrm{~mm}$, width $B=25.4 \mathrm{~mm}$, and thickness $H=2 h=3 \mathrm{~mm}$; the initial delamination length is $a=32 \mathrm{~mm}$. The elastic moduli of the material are $E_{x}=129 \mathrm{GPa}, E_{y}=E_{z}=10.1 \mathrm{GPa}$, and $G_{x y}=G_{z x}=5.5 \mathrm{GPa}$.

Apart from the purposes of comparison, the finite element model has also been exploited to numerically implement a compliance calibration strategy, which enables accurate estimation of the values of the interface elastic constants to be used in applying the EBT model. As a first step, two analyses corresponding to a DCB (Fig. 5b) and an ENF (Fig. 5c) test have been performed, and the corresponding values of compliance, $C_{\mathrm{DCB}}^{\mathrm{FEM}}$ and $C_{\mathrm{ENF}}^{\mathrm{FEM}}$, computed. Then, these values were made equal to the analytical predictions, $C_{\mathrm{DCB}}^{\mathrm{EBT}}$ and $C_{\mathrm{ENF}}^{\mathrm{EBT}}$, given by Eqs. (35), which were numerically solved to yield $k_{x}$ and $k_{z}$. For the specimen under examination, the numerical analyses yielded $C_{\mathrm{DCB}}^{\mathrm{FEM}}=0.0301 \mathrm{~mm} / \mathrm{N}$ and $C_{\mathrm{ENF}}^{\mathrm{FEM}}=0.0041 \mathrm{~mm} / \mathrm{N}$, whence the constants $k_{x}=31550 \mathrm{~N} / \mathrm{mm}^{3}$ and $k_{z}=23150 \mathrm{~N} / \mathrm{mm}^{3}$ were then determined. The strategy is illustrated in figures $6 \mathrm{a}$ and $6 \mathrm{~b}$, where $C_{\mathrm{DCB}}^{\mathrm{EBT}}$ and $C_{\mathrm{ENF}}^{\mathrm{EBT}}$ are plotted as functions of $k_{z}$ and $k_{x}$, respectively. Section 6 of this paper describes how this compliance calibration strategy can be applied to experimental results. 


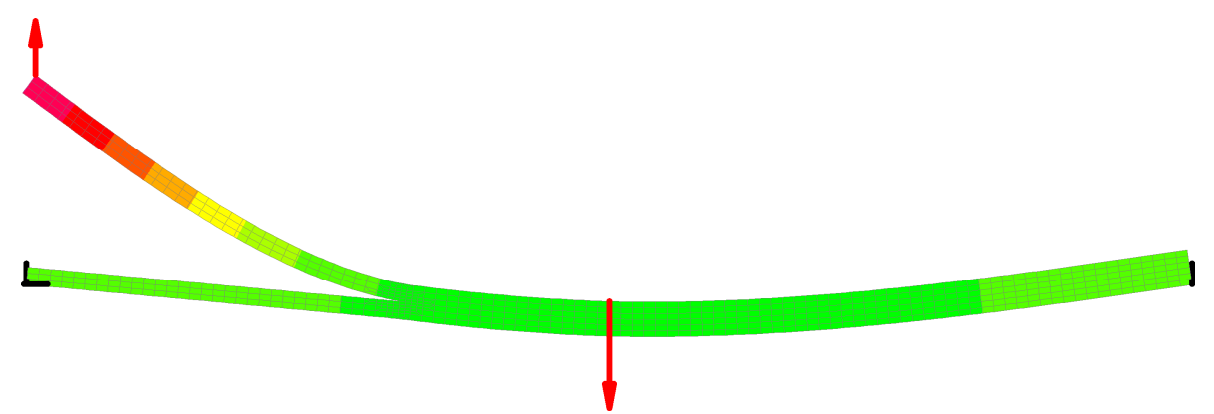

(a)

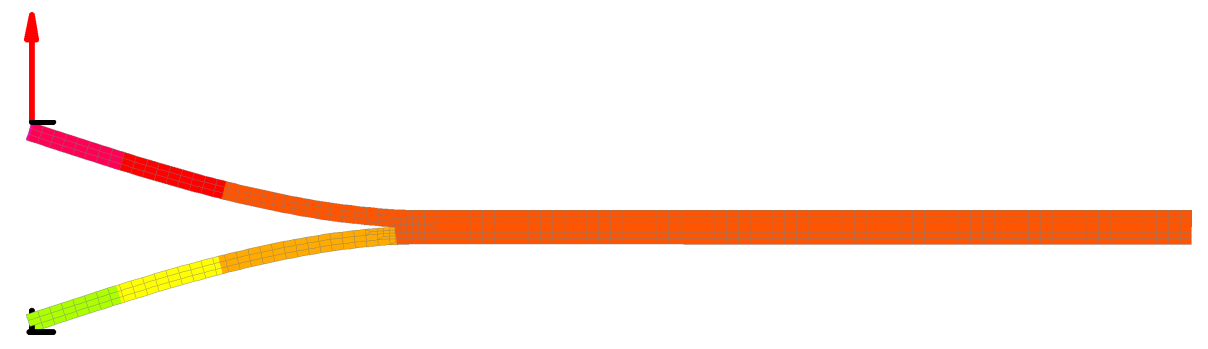

(b)

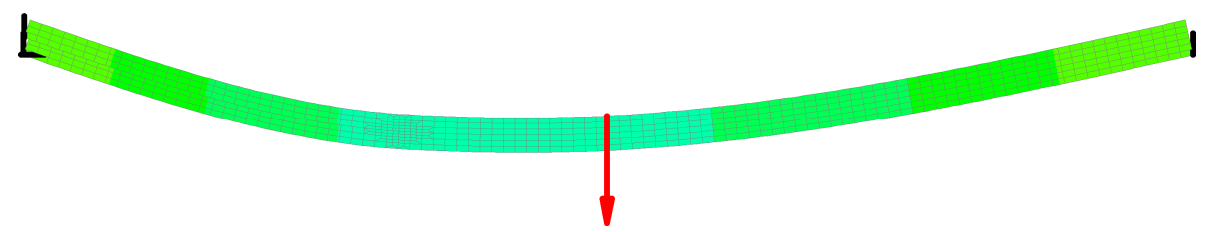

(c)

Plate Disp $(\mathrm{mm})$

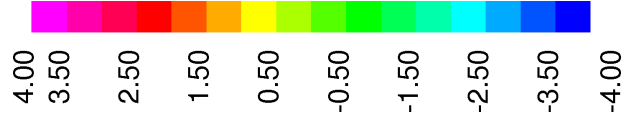

Fig. 5 Finite element model: (a) MMB test; (b) DCB test; (c) ENF test (displacements are exaggerated for clarity)

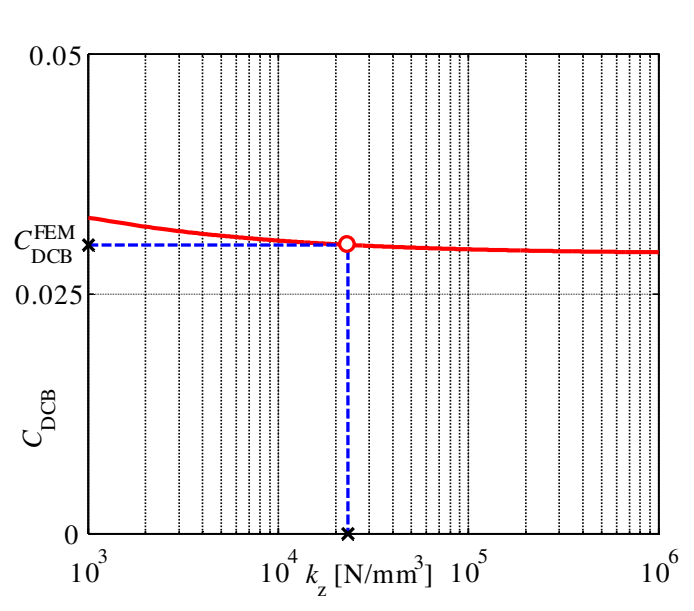

(a)

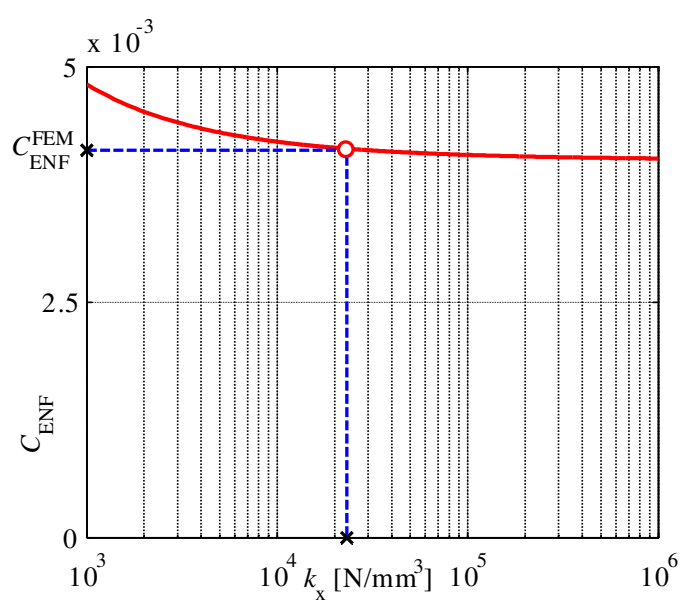

(b)

Fig. 6 Compliance calibration strategy: (a) determination of $k_{z}$; (b) determination of $k_{x}$ 


\subsection{Comparison}

The analytical models presented in the foregoing are now compared with each other and with the finite element model, with specific regard to their predictions of the compliance.

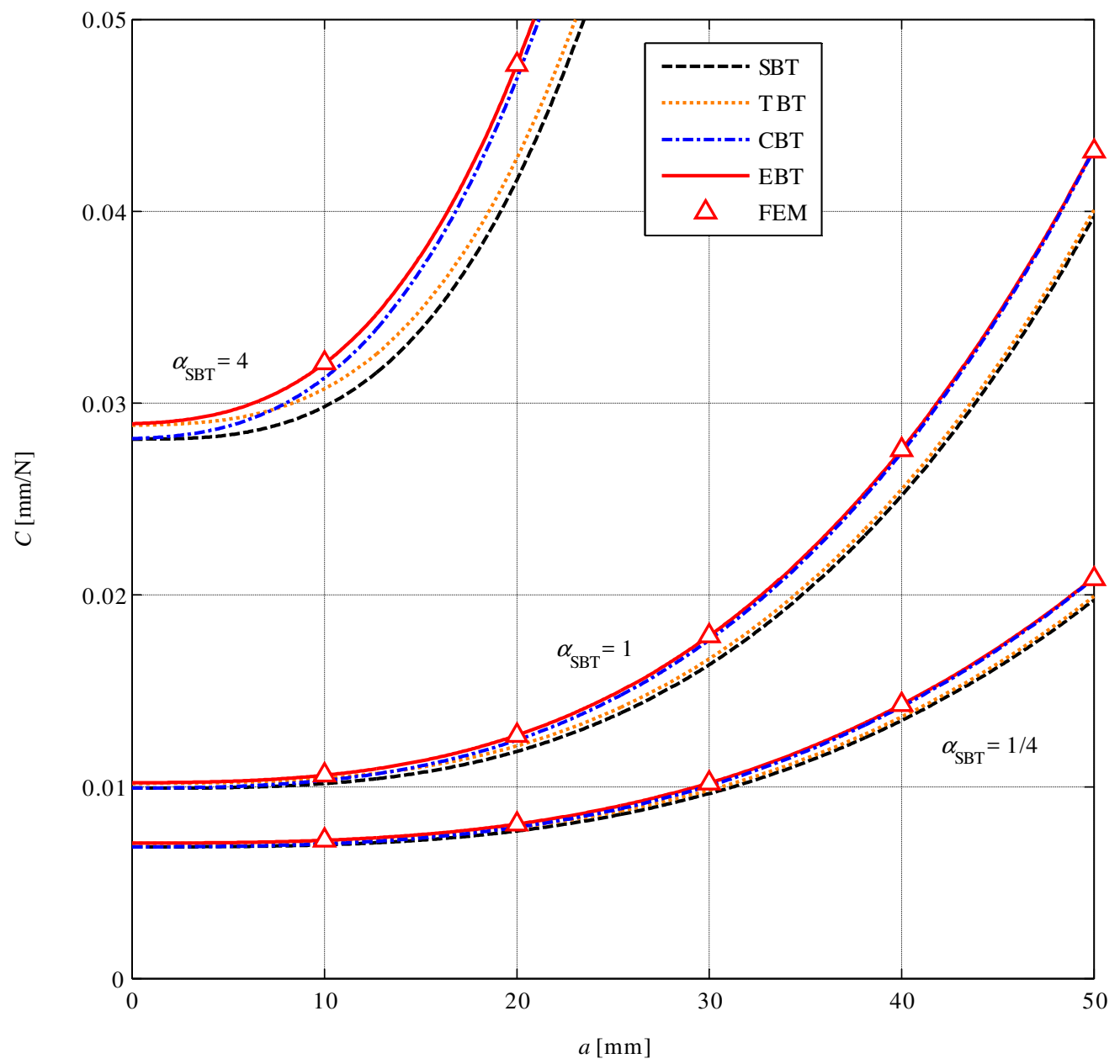

Fig. 7 Compliance vs. delamination length

Figure 7 shows the compliance, $C$, as a function of the delamination length, $a$, as predicted by the SBT, TBT, CBT, and EBT models. The compliance values computed via the finite element analysis for discrete values of delamination length are also shown. The same plot shows three values $(1 / 4,1$, and 4$)$ of the SBT mixed-mode ratio, $\alpha_{\mathrm{SBT}}=G_{\mathrm{I}}^{\mathrm{SBT}} / G_{\mathrm{II}}^{\mathrm{SBT}}$ (see Section 5). The SBT model markedly underestimates the compliance with respect to the finite element analysis. The TBT model yields better predictions, especially for short delamination lengths, when the delaminated sublaminates behave as 'thick' beams. The CBT model improves on the predictions of the SBT model, but still underestimates the finite 
element results because it does not consider the sublaminates' shear deformation appropriately. Only the EBT model takes into account both the local deformation at the crack tip and shear deformation of the sublaminates, thus closely matching the finite element results throughout the entire range of delamination lengths.

\section{Energy release rate}

\subsection{Definition}

The energy release rate is defined as $G=-d V / d A$, where $V$ is the total potential energy of the system and $d A=B d a$ is the area of the new surface created by crack advancement [6]. For a linear mechanical model,

$G=\frac{P^{2}}{2 B} \frac{d C}{d a}$

where suitable expressions or values of $C$ should be used, depending on the mechanical model adopted.

Under I/II mixed-mode fracture conditions, the energy release rate, $G$, is the sum of two contributions, $G_{\mathrm{I}}$ and $G_{\mathrm{II}}$, related to fracture modes I and II, respectively. Several alternative, albeit non-equivalent, methods have been proposed to compute the modal contributions to the energy release rate in general delamination fracture problems [26]. For a symmetric MMB test specimen, however, the mode I and II contributions to the energy release rate can be obtained simply by regarding the test as the superposition of DCB and ENF tests. Within the EBT model, this approach is equivalent to computing $G_{\text {I }}$ and $G_{\text {II }}$ based on the peak values of the interfacial stresses at the crack tip, which is the general method to be used in the case of asymmetric delaminations [20].

Substituting Eq. (10) into (36), and observing that from Eqs. (7) $P_{\mathrm{I}} / P$ and $P_{\mathrm{II}} / P$ are independent of $a$, we obtain

$G=G_{\mathrm{I}}+G_{\mathrm{II}}$,

where

$G_{\mathrm{I}}=\frac{P_{\mathrm{I}}^{2}}{2 B} \frac{d C_{\mathrm{DCB}}}{d a}$ and $G_{\mathrm{II}}=\frac{P_{\mathrm{II}}^{2}}{2 B} \frac{d C_{\mathrm{ENF}}}{d a}$. 
Note that in the first of Eqs. (38) only positive values of $P_{\mathrm{I}}$ should be considered, as negative values produce compressive normal stresses at the crack tip and closure of the crack faces. Thus, for $P_{\mathrm{I}} \leq 0$ the mode I contribution is $G_{\text {I }}=0$.

\subsection{Beam-theory models}

In the SBT model $[2,3]$, the expressions for the modal contributions to the energy release rate are obtained by substituting Eqs. (13) into (38),

$G_{\mathrm{I}}^{\mathrm{SBT}}=\frac{P_{\mathrm{I}}^{2} a^{2}}{B^{2} D_{1}} \quad$ and $\quad G_{\mathrm{II}}^{\mathrm{SBT}}=\frac{P_{\mathrm{II}}^{2} a^{2}}{16 B^{2} D_{1}} \frac{A_{1} h^{2}}{A_{1} h^{2}+4 D_{1}}$.

In particular, for orthotropic specimens, from Eqs. (15) we have

$G_{\mathrm{I}}^{\mathrm{SBT}}=\frac{12 P_{\mathrm{I}}^{2} a^{2}}{B^{2} E_{x} h^{3}} \quad$ and $\quad G_{\mathrm{II}}^{\mathrm{SBT}}=\frac{9 P_{\mathrm{II}}^{2} a^{2}}{16 B^{2} E_{x} h^{3}}$.

Likewise, in the TBT model, the modal contributions to the energy release rate are obtained by substituting Eqs. (17) into (38),

$G_{\mathrm{I}}^{\mathrm{TBT}}=\frac{P_{\mathrm{I}}^{2}}{B^{2}}\left(\frac{a^{2}}{D_{1}}+\frac{1}{C_{1}}\right) \quad$ and $\quad G_{\mathrm{II}}^{\mathrm{TBT}}=\frac{P_{\mathrm{II}}^{2} a^{2}}{16 B^{2} D_{1}} \frac{A_{1} h^{2}}{A_{1} h^{2}+4 D_{1}}=G_{\mathrm{II}}^{\mathrm{SBT}}$.

It is interesting to note that transverse shear deformability does not influence the mode II contribution to the energy release rate. This is not surprising considering that the correction term for $C_{\mathrm{ENF}}^{\mathrm{TBT}}$ in Eq. (17) is independent of $a$. Nevertheless, a shear correction term for $G_{I I}$ is commonly given in the literature [6]. This shear correction term, introduced for the ENF test specimen by Carlsson et al. [27] and later uncritically reported by a number of authors, has recently been proved incorrect by Fan et al. [28] (see also Valvo [29] for a more detailed discussion of this topic).

\subsection{Corrected beam-theory model}

Now, introducing the increased crack lengths (19) into Eqs. (40) yields the CBT model expressions for the energy release rate contributions [8],

$$
G_{\mathrm{I}}^{\mathrm{CBT}}=\frac{12 P_{\mathrm{I}}^{2}}{B^{2} E_{x} h^{3}}\left(a+\chi_{\mathrm{I}} h\right)^{2} \quad \text { and } \quad G_{\mathrm{II}}^{\mathrm{CBT}}=\frac{9 P_{\mathrm{II}}^{2}}{16 B^{2} E_{x} h^{3}}\left(a+\chi_{\mathrm{II}} h\right)^{2},
$$


where $\chi_{\mathrm{I}}$ and $\chi_{\text {II }}$ are given by Eqs. (20). In an effort to extend the CBT model to MD laminates, the crack length correction factors can be introduced into Eqs. (39), yielding

$G_{\mathrm{I}}^{\mathrm{CBT}}=\frac{P_{\mathrm{I}}^{2}}{B^{2} D_{1}}\left(a+\chi_{\mathrm{I}} h\right)^{2} \quad$ and $\quad G_{\mathrm{II}}^{\mathrm{CBT}}=\frac{P_{\mathrm{II}}^{2}}{16 B^{2} D_{1}} \frac{A_{1} h^{2}}{A_{1} h^{2}+4 D_{1}}\left(a+\chi_{\mathrm{II}} h\right)^{2}$,

where, however, Eqs. (20) cannot be used to compute $\chi_{\mathrm{I}}$ and $\chi_{\mathrm{II}}$, and the same considerations reported at the end of Section 3.3 apply.

\subsection{Enhanced beam-theory model}

In the EBT model, the mode I and II contributions to $G$ can be computed from [20]

$G_{\mathrm{I}}^{\mathrm{EBT}}=\frac{\sigma_{0}^{2}}{2 k_{z}} \quad$ and $\quad G_{\mathrm{II}}^{\mathrm{EBT}}=\frac{\tau_{0}^{2}}{2 k_{x}}$,

where $\sigma_{0}$ and $\tau_{0}$ are respectively the normal and tangential interfacial stresses at the crack tip (note that only positive tensile $\sigma_{0}$ contribute to $G_{\mathrm{I}}$, hence $\sigma_{0}<0$ implies $G_{\mathrm{I}}=0$ ). By recalling Eqs. (36) and (52) in Part I [7], we find

$$
\begin{aligned}
\sigma_{0}= & \left.\sigma\right|_{s=0}=\frac{P_{\mathrm{I}}}{B}\left[\frac{\left(\lambda_{1}^{2}-\lambda_{2}^{2}\right)\left(\lambda_{1} \tanh \lambda_{2} b-\lambda_{2} \tanh \lambda_{1} b\right)}{\left(\lambda_{1}^{2}+\lambda_{2}^{2}\right) \tanh \lambda_{1} b \tanh \lambda_{2} b-2 \lambda_{1} \lambda_{2}\left(1-\operatorname{sech} \lambda_{1} b \operatorname{sech} \lambda_{2} b\right)}+\right. \\
& +\lambda_{1} \lambda_{2} a \frac{\left(\lambda_{1}^{2}+\lambda_{2}^{2}\right)\left(1-\operatorname{sech} \lambda_{1} b \operatorname{sech} \lambda_{2} b\right)-2 \lambda_{1} \lambda_{2} \tanh \lambda_{1} b \tanh \lambda_{2} b}{\left(\lambda_{1}^{2}+\lambda_{2}^{2}\right) \tanh \lambda_{1} b \tanh \lambda_{2} b-2 \lambda_{1} \lambda_{2}\left(1-\operatorname{sech} \lambda_{1} b \operatorname{sech} \lambda_{2} b\right)} \\
\tau_{0}=\left.\tau\right|_{s=0}= & \frac{P_{\mathrm{II}}}{B h} \frac{A_{1} h^{2}}{A_{1} h^{2}+4 D_{1}}\left[\frac{1}{2}\left(1+\lambda_{5} a \operatorname{coth} \lambda_{5} b\right)-\frac{\sinh \lambda_{5} \ell}{\sinh \lambda_{5} b}\right] .
\end{aligned}
$$

Now, by substituting Eqs. (45) into (44), the modal contributions to the energy release rate are obtained as

$$
G_{\mathrm{I}}^{\mathrm{EBT}}=\mu_{\mathrm{I}} G_{\mathrm{I}}^{\mathrm{SBT}} \quad \text { and } \quad G_{\mathrm{II}}^{\mathrm{EBT}}=\mu_{\mathrm{II}} G_{\mathrm{II}}^{\mathrm{SBT}},
$$

where

$$
\begin{aligned}
\mu_{\mathrm{I}}= & {\left[\frac{\left(\lambda_{1}^{2}+\lambda_{2}^{2}\right)\left(1-\operatorname{sech} \lambda_{1} b \operatorname{sech} \lambda_{2} b\right)-2 \lambda_{1} \lambda_{2} \tanh \lambda_{1} b \tanh \lambda_{2} b}{\left(\lambda_{1}^{2}+\lambda_{2}^{2}\right) \tanh \lambda_{1} b \tanh \lambda_{2} b-2 \lambda_{1} \lambda_{2}\left(1-\operatorname{sech} \lambda_{1} b \operatorname{sech} \lambda_{2} b\right)}+\right.} \\
& \left.+\frac{1}{a} \frac{\lambda_{1}^{2}-\lambda_{2}^{2}}{\lambda_{1} \lambda_{2}} \frac{\lambda_{1} \tanh \lambda_{2} b-\lambda_{2} \tanh \lambda_{1} b}{\left(\lambda_{1}^{2}+\lambda_{2}^{2}\right) \tanh \lambda_{1} b \tanh \lambda_{2} b-2 \lambda_{1} \lambda_{2}\left(1-\operatorname{sech} \lambda_{1} b \operatorname{sech} \lambda_{2} b\right)}\right]^{2},
\end{aligned}
$$


$\mu_{\mathrm{II}}=\left[\operatorname{coth} \lambda_{5} b+\frac{1}{\lambda_{5} a}\left(1-2 \frac{\sinh \lambda_{5} \ell}{\sinh \lambda_{5} b}\right)\right]^{2}$

are correction factors with respect to the SBT model.

Figure 8 shows in semi-logarithmic scale the mode I and II correction factors, $\mu_{\mathrm{I}}$ and $\mu_{\mathrm{II}}$, as functions of the interface elastic constants, $k_{z}$ and $k_{x}$, respectively. The blue, dashed curves in figure 8 a show how $\mu_{\mathrm{I}}$ (hence $G_{\mathrm{I}}^{\mathrm{EBT}}$ ) decreases with increasing $C_{1}$. Instead, $\mu_{\mathrm{II}}$ (hence $G_{\mathrm{II}}^{\mathrm{EBT}}$ ) turns out to be independent of the sublaminates' shear deformability, as in the TBT model. Both correction factors are decreasing functions of the elastic constants and approach finite limits as the elastic constants go to infinity. The circles in figure 8 denote points corresponding to the values of the elastic constants estimated through the numerical compliance calibration strategy described in Section 3.5, for which $\mu_{\mathrm{I}}=1.086$ and $\mu_{\mathrm{II}}=1.056$. As apparent from the enlarged views of the plots, both $\mu_{\mathrm{I}}$ and $\mu_{\mathrm{II}}$ depend only weakly on the interface constants in rather wide ranges close to the estimated values. Thus, presumably, even rough estimates of the interface constants may enable determination of $G_{\mathrm{I}}^{\mathrm{EBT}}$ and $G_{\mathrm{II}}^{\mathrm{EBT}}$ with acceptable precision in many cases. For instance, following Corigliano [30],

$k_{x} \cong \frac{2 G_{z x}}{e}$ and $\quad k_{z} \cong \frac{E_{z}}{e}$,

where $e$ is a fictitious thickness about one order of magnitude smaller than the laminate's thickness. If we assume $e=2 h / 10$, from (48) we obtain $k_{x} \cong 36666 \mathrm{~N} / \mathrm{mm}^{3}$ and $k_{z} \cong 33666 \mathrm{~N} / \mathrm{mm}^{3}$. The EBT model in this case furnishes $\mu_{\mathrm{I}} \cong 1.079$ and $\mu_{\mathrm{II}} \cong 1.051$, which deviate by less than $1 \%$ from the values corresponding to the accurate estimates of the interface constants. The points corresponding to the correction factors computed using Eqs. (48) are represented by the triangles in figure 8 . 


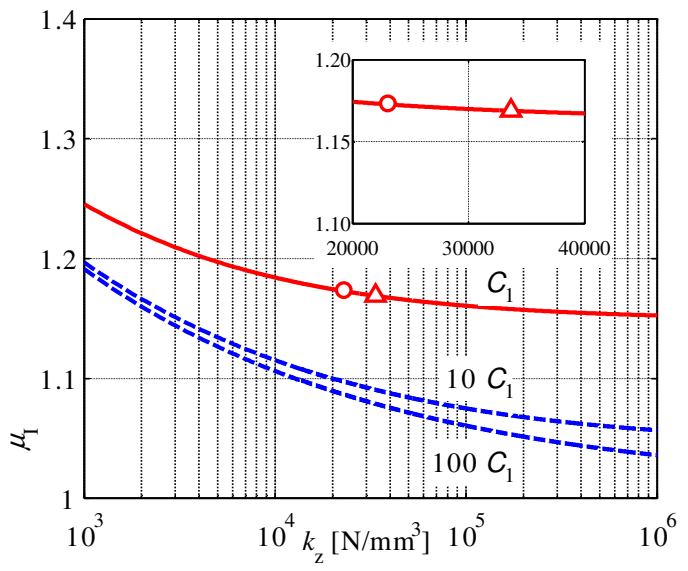

(a)

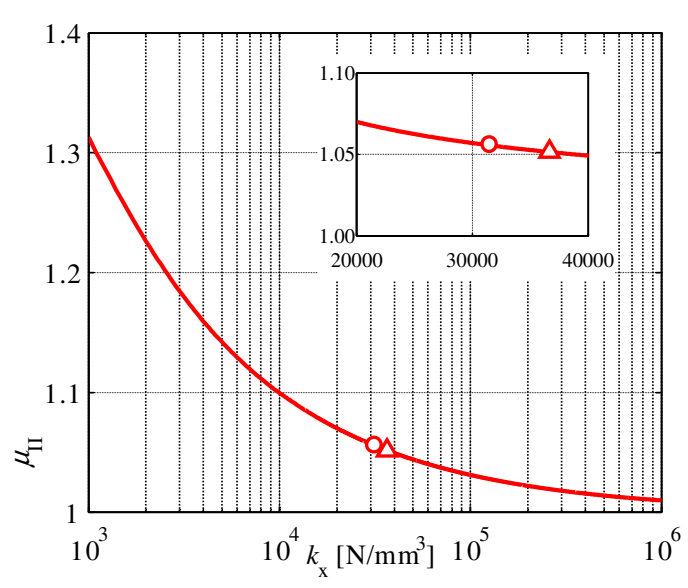

(b)

Fig. 8 energy release rate correction factors vs. interface elastic constants: (a) mode I; (b) mode II

In practice, instead of using the exact but complicated Eqs. (46-47), the previously adopted simplifying assumptions (namely, that the arguments of the hyperbolic functions are very large) enable using the following approximate expressions for the modal contributions to the energy release rate without significant loss of accuracy:

$G_{\mathrm{I}}^{\mathrm{EBT}} \cong \frac{P_{\mathrm{I}}^{2}}{B^{2} D_{1}}\left(a+\frac{1}{\lambda_{1}}+\frac{1}{\lambda_{2}}\right)^{2} \quad$ and $\quad G_{\mathrm{II}}^{\mathrm{EBT}} \cong \frac{P_{\mathrm{II}}^{2}}{16 B^{2} D_{1}} \frac{A_{1} h^{2}}{A_{1} h^{2}+4 D_{1}}\left(a+\frac{1}{\lambda_{5}}\right)^{2}$,

and for orthotropic specimens

$G_{\mathrm{I}}^{\mathrm{EBT}} \cong \frac{12 P_{\mathrm{I}}^{2}}{B^{2} E_{x} h^{3}}\left(a+\frac{1}{\lambda_{1}}+\frac{1}{\lambda_{2}}\right)^{2} \quad$ and $\quad G_{\mathrm{II}}^{\mathrm{EBT}} \cong \frac{12 P_{\mathrm{II}}^{2}}{B^{2} E_{x} h^{3}}\left(a+\frac{1}{\lambda_{5}}\right)^{2}$.

Expressions similar to Eqs. (50) have been given for the mode I contribution by Reeder and Crews [2] and for the mode II contribution by Wang and Qiao [13], de Morais [14] and Jumel et al. [15]. Moreover, Budzik et al. have furnished an experimental validation and highlighted the correlation between $1 / \lambda_{5}$ and the length of the process zone in mode II fracture [31]. Likewise, $1 / \lambda_{1}+1 / \lambda_{2}$ can be interpreted as the length of the process zone in mode I fracture. We can shed further light on the role played by these parameters by considering the limit case of a rigid interface, for which

$\lim _{k_{z} \rightarrow \infty} \frac{1}{\lambda_{1}}=0, \quad \lim _{k_{z} \rightarrow \infty} \frac{1}{\lambda_{2}}=\sqrt{\frac{D_{1}}{C_{1}}}, \quad$ and $\quad \lim _{k_{x} \rightarrow \infty} \frac{1}{\lambda_{5}}=0$ 
Note that by substituting Eqs. (51) into (49), we retrieve Eqs. (41) of the TBT model, except for an additional term in the mode I contribution, which accounts for the different rotations of the upper and lower sublaminates' cross sections. In fact, though connected by a rigid interface, the two sublaminates behave as a 'monolithic' beam only for $C_{1} \rightarrow \infty$, in which case the EBT model reduces to the SBT model.

By comparing Eqs. (49) with (43), we obtain crack length correction parameters analogous to those introduced in the CBT model:

$$
\chi_{\mathrm{I}}^{\mathrm{EBT}}=\frac{1}{h}\left(\frac{1}{\lambda_{1}}+\frac{1}{\lambda_{2}}\right)=\frac{1}{h} \sqrt{\frac{D_{1}}{C_{1}}+\sqrt{\frac{2 D_{1}}{k_{z}}}} \text { and } \quad \chi_{\mathrm{II}}^{\mathrm{EBT}}=\frac{1}{h} \frac{1}{\lambda_{5}}=\frac{1}{h} \frac{1}{\left.\sqrt{2 k_{x}\left(\frac{1}{A_{1}}+\frac{h^{2}}{4 D_{1}}\right.}\right)} .
$$

Eqs. (52) offer the significant advantage that they can be used for generic laminated specimens, not only orthotropic specimens, for which they become

$$
\chi_{\mathrm{I}}^{\mathrm{EBT}}=\sqrt{\frac{E_{x}}{10 G_{z x}}+\sqrt{\frac{E_{x}}{6 k_{z} h}}} \text { and } \quad \chi_{\mathrm{II}}^{\mathrm{EBT}}=\sqrt{\frac{E_{x}}{8 k_{x} h}} .
$$

With the estimates of the interface elastic constants given by Eqs. (48), we obtain

$$
\chi_{\mathrm{I}}^{\mathrm{EBT}} \cong \sqrt{\frac{E_{x}}{10 G_{z x}}+\sqrt{\frac{E_{x}}{6 E_{z}} \frac{e}{h}}} \text { and } \chi_{\mathrm{II}}^{\mathrm{EBT}} \cong \sqrt{\frac{E_{x}}{16 G_{z x}} \frac{e}{h}}
$$

Figure 9 shows a plot of the crack length correction parameters computed according to the CBT and EBT models - through Eqs. (20) and (54), respectively - as functions of the elastic moduli of the laminate. Comparison shows excellent agreement for $\chi_{\mathrm{I}}$, despite the apparently different analytical expressions. Higher discrepancies can be observed for $\chi_{\text {II }}$, for which we furnish a distinct definition, while the CBT model simply considers it a fraction of $\chi_{\mathrm{I}}$. It is worth noting that according to the EBT model $\chi_{\text {II }}$ does not depend on $E_{z}$. The circles in figure 9 denote points corresponding to the elastic constants of the UD laminated specimen given in Section 3.5. 


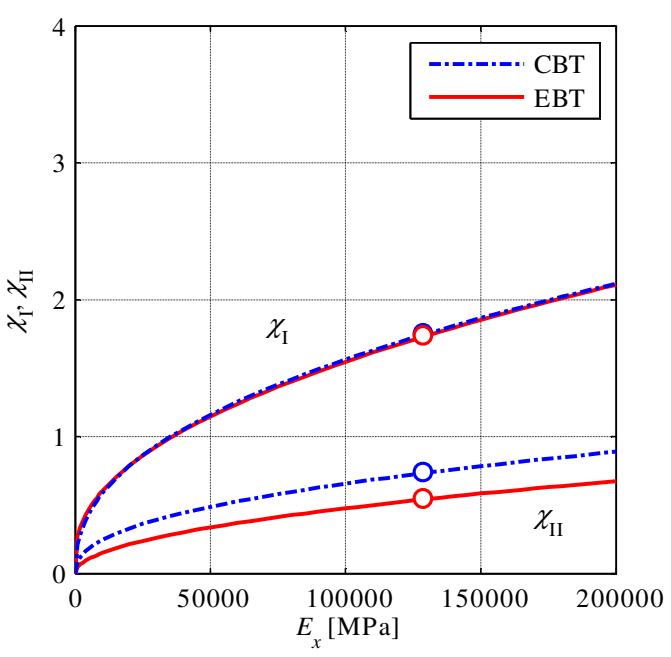

(a)

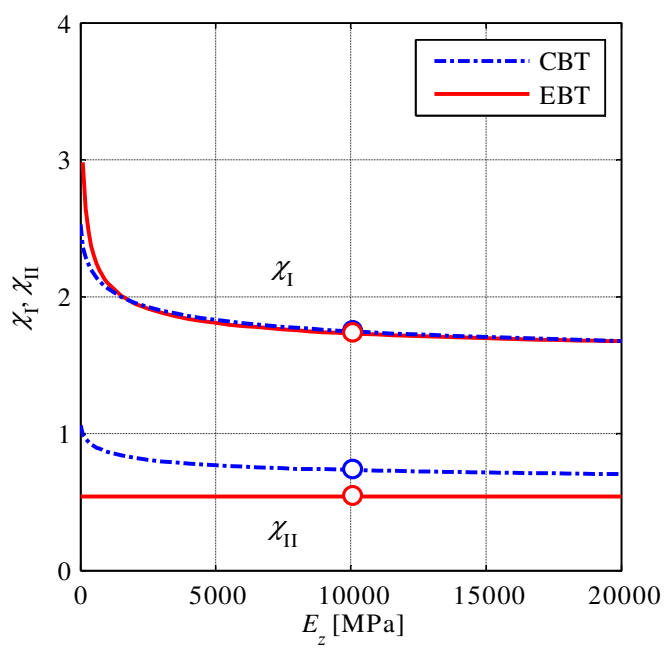

(b)

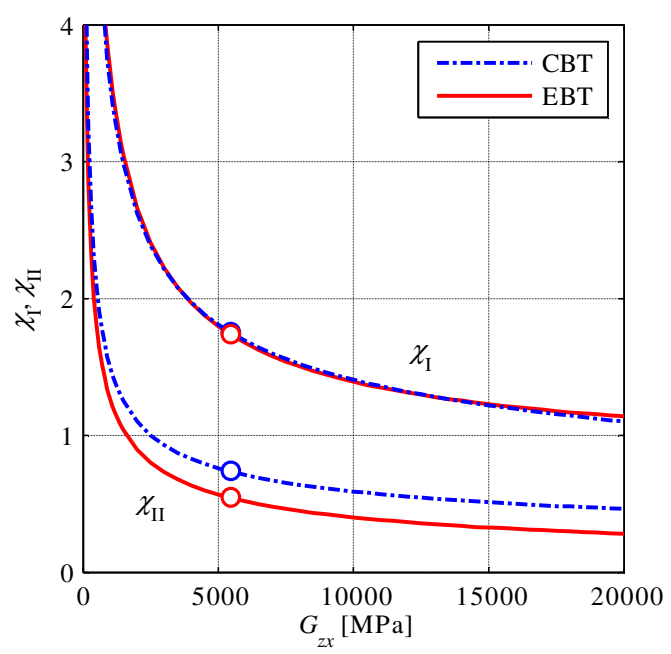

(c)

Fig. 9 Crack length correction parameters vs. elastic moduli: (a) longitudinal Young's modulus;

(b) transverse Young's modulus; (c) shear modulus

\subsection{Comparison}

In order to compare the analytical models presented in previous Sections with each other, we consider an MMB test specimen having the geometrical and mechanical properties listed in Section 3.5.

Figure 10a shows the energy release rate, $\mathrm{G}$, as a function of the delamination length, a, as predicted by the SBT, TBT, CBT, and EBT models. Similarly, figures $10 \mathrm{~b}$ and $10 \mathrm{c}$ show the mode I and II contributions, $G_{\mathrm{I}}$ and $G_{\mathrm{II}}$, respectively. Three values $(1 / 4,1$, and 4$)$ of the SBT mixed-mode ratio, $\alpha_{\mathrm{SBT}}=G_{\mathrm{I}}^{\mathrm{SBT}} / G_{\mathrm{II}}^{\mathrm{SBT}}$, have been considered. For all models compared, the energy release rate and its modal contributions appear as increasing functions of the 
delamination length. Both the SBT and TBT models underestimate the energy release rate with respect to the $\mathrm{CBT}$ and $\mathrm{EBT}$ models. The latter two models are for the most part in agreement, except for very large values of $a$ (i.e. when the delamination crack tip approaches the downward load application point), for which the EBT model shows some boundary effects related to the mode II contribution. These boundary effects disappear if the approximate Eqs. (49) are used instead of the exact Eqs. (46)-(47).

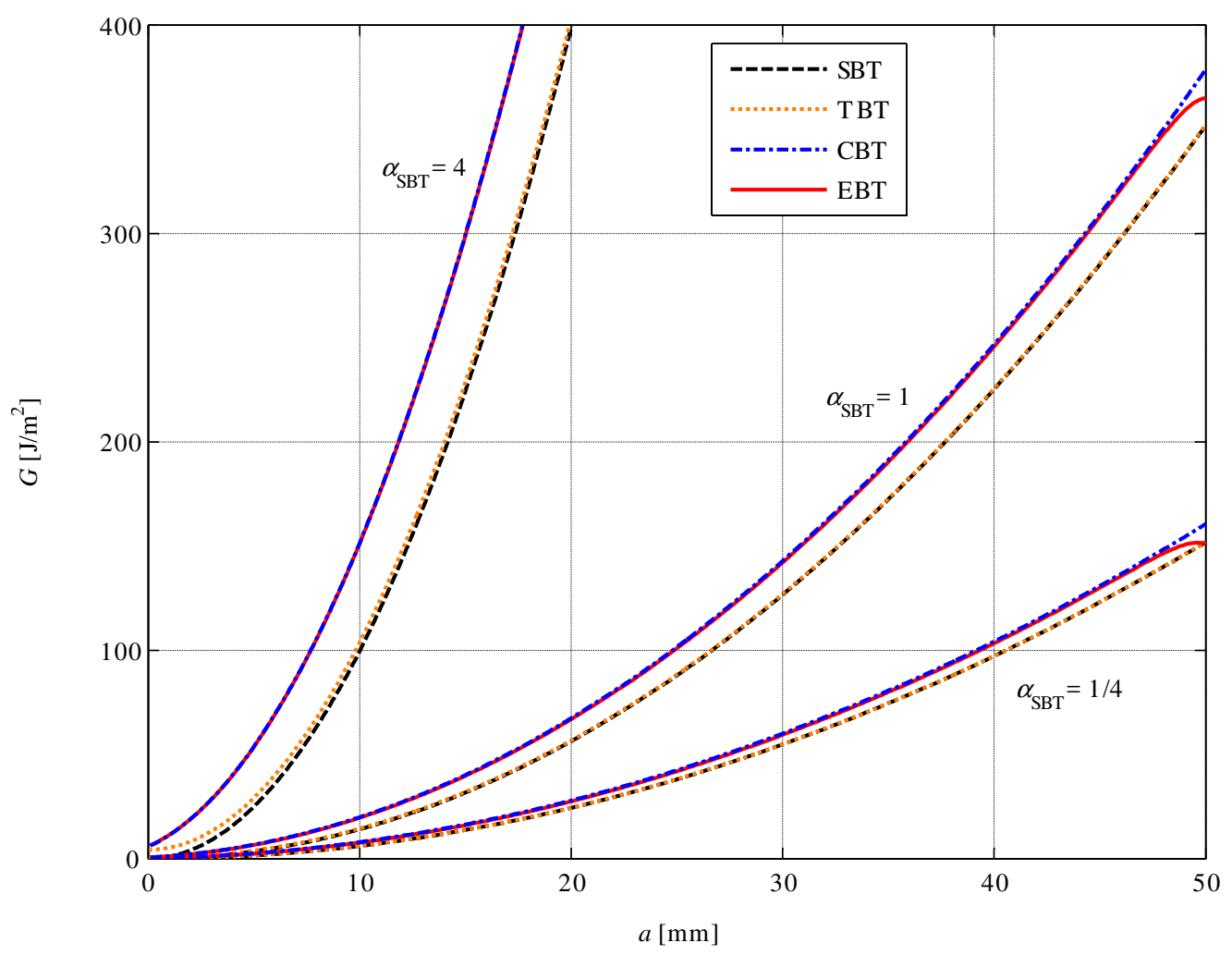

(a)

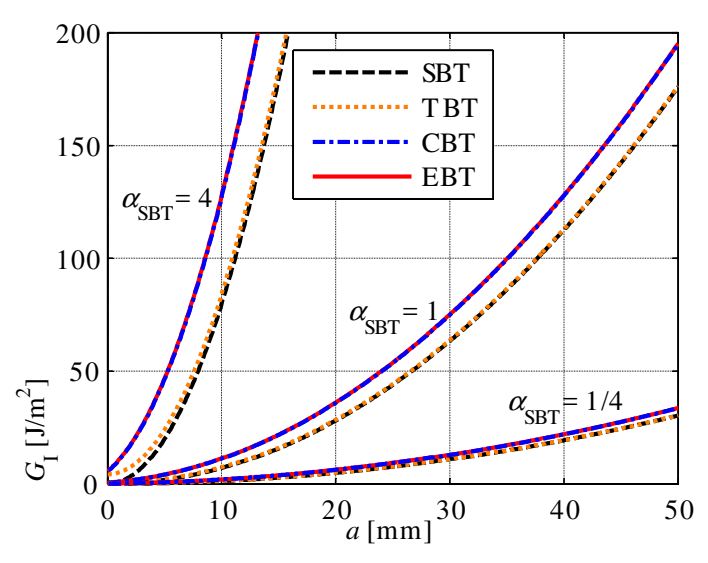

(b)

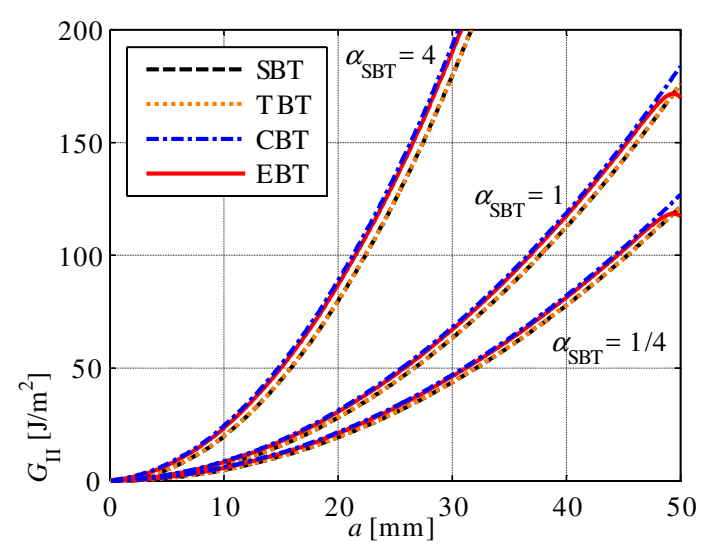

(c)

Fig. 10 Energy release rate vs. delamination length: (a) total; (b) mode I; (c) mode II 
Table 1 Relationships between alternative measures of mode mixity

\begin{tabular}{ccccc}
\hline & $\gamma_{\mathrm{I}}$ & $\gamma_{\mathrm{II}}$ & $\alpha$ & $\psi$ \\
\hline$\gamma_{\mathrm{I}}$ & - & $1-\gamma_{\mathrm{II}}$ & $\frac{1}{1+1 / \alpha}$ & $\cos ^{2} \psi$ \\
\hline$\gamma_{\mathrm{II}}$ & $1-\gamma_{\mathrm{I}}$ & - & $\frac{1}{1+\alpha}$ & $\sin ^{2} \psi$ \\
\hline$\alpha$ & $\frac{1}{1 / \gamma_{\mathrm{I}}-1}$ & $1 / \gamma_{\mathrm{II}}-1$ & - & $\cot ^{2} \psi$ \\
\hline$\psi$ & $\arctan \sqrt{1 / \gamma_{\mathrm{I}}-1}$ & $\arctan \frac{1}{\sqrt{1 / \gamma_{\mathrm{II}}-1}}$ & $\arctan \frac{1}{\sqrt{\alpha}}$ & - \\
\hline
\end{tabular}

\section{$5 \quad$ Mode mixity}

Under I/II mixed-mode fracture conditions, the relative contributions of the two fracture modes are characterised through a conventional measure of mode mixity [26]. In the MMB test, the mode mixity is usually specified through the I/II mixed-mode ratio [5],

$\alpha=\frac{G_{\mathrm{I}}}{G_{\mathrm{II}}}=\frac{\gamma_{\mathrm{I}}}{\gamma_{\mathrm{II}}}$

where

$\gamma_{\mathrm{I}}=\frac{G_{\mathrm{I}}}{G} \quad$ and $\quad \gamma_{\mathrm{II}}=\frac{G_{\mathrm{II}}}{G}$

are the relative mode I and II contributions to the energy release rate, which, recalling Eq. (31), satisfy $\gamma_{\mathrm{I}}+\gamma_{\mathrm{II}}=1$. Since the energy release rate contributions are positive quantities, we may put $\gamma_{\mathrm{I}}=\cos ^{2} \psi$ and $\gamma_{\mathrm{II}}=\sin ^{2} \psi$, where

$\psi=\arctan \sqrt{\frac{\gamma_{\mathrm{II}}}{\gamma_{\mathrm{I}}}}=\arctan \sqrt{\frac{G_{\mathrm{II}}}{G_{\mathrm{I}}}}$

is the mode-mixity angle [26]. The relationships between alternative measures of mode mixity are given in Table 1 .

From Eqs. (46) and recalling Eqs. (7) and (39), the mixed-mode ratio predicted by the EBT model for the MMB test specimen is

$$
\alpha=\frac{\mu_{\mathrm{I}} G_{\mathrm{I}}^{\mathrm{SBT}}}{\mu_{\mathrm{II}} G_{\mathrm{II}}^{\mathrm{SBT}}}=\frac{\mu_{\mathrm{I}}}{\mu_{\mathrm{II}}}\left(1+\frac{4 D_{1}}{A_{1} h^{2}}\right)\left(\frac{3 c-\ell}{c+\ell}\right)^{2} .
$$


Eq. (58) can be solved with respect to $c$ to obtain the lever arm length value to use in tests to obtain a desired value of the mode mixity,

$$
c=\frac{\beta \sqrt{1+\frac{4 D_{1}}{A_{1} h^{2}}}+\sqrt{\alpha}}{3 \beta \sqrt{1+\frac{4 D_{1}}{A_{1} h^{2}}}-\sqrt{\alpha}} .
$$

where

$$
\beta=\frac{a+\chi_{\mathrm{I}} h}{a+\chi_{\mathrm{II}} h}=\sqrt{\frac{\mu_{\mathrm{I}}}{\mu_{\mathrm{II}}}} \cong \frac{a+1 / \lambda_{1}+1 / \lambda_{2}}{a+1 / \lambda_{5}} .
$$

In particular, for orthotropic specimens, we have

$$
c=\frac{2 \beta+\sqrt{3 \alpha}}{6 \beta-\sqrt{3 \alpha}} \ell
$$

which is equivalent to Eq. (5) of the ASTM standard [5].

By inspecting Eq. (58), it can be deduced that pure mode I fracture $(\alpha \rightarrow \infty)$ would require a lever arm length $c=-\ell$, together with a negative value of load $P$ (see Eqs. (3)). Such conditions cannot however occur in practice, hence pure mode I tests cannot be performed using MMB testing equipment. Conversely, pure mode II fracture $(\alpha=0)$ can be obtained by choosing $c=\ell / 3$. It is worthwhile noting that lever arm lengths below such value also result in pure mode II fracture because the mode I load acts by closing the delamination crack instead of opening it. Actually, Eq. (7) yields negative values of $P_{\mathrm{I}}$, hence there is no mode I contribution to the energy release rate. In experimental practice [2, 3, 6], pure mode II is often obtained by setting $c=0$, though the measured fracture toughness may be overestimated because of contact and friction between the specimen's two arms [23].

Since the lever arm lengths are fixed during any particular test, the mode mixity computed according to the SBT model does not change with crack propagation, hence the MMB test is commonly referred to as a constant mixedmode fracture test. However, it is a well-known fact that if a more complex model is used to interpret test results, the mode mixity turns out to be dependent on delamination length and differs slightly from the 'nominal' value predicted by the SBT model. Figure 11 shows the mixed-mode ratio, $\alpha$, as a function of the 
delamination length, $a$, as computed according to the SBT, TBT, CBT, and EBT models. The SBT model underestimates $\alpha$ with respect to the other models. The TBT model is mostly in agreement with the SBT model, except for short delamination lengths, when the delaminated sublaminates behave as 'thick' beams. The CBT and EBT models yield similar predictions, which differ from those of the SBT and TBT models especially in the case of prevailing mode I fracture. Indeed, this is when the crack-tip relative displacements and rotations not taken into account by the simpler models - exert the utmost influence on the specimen's response.

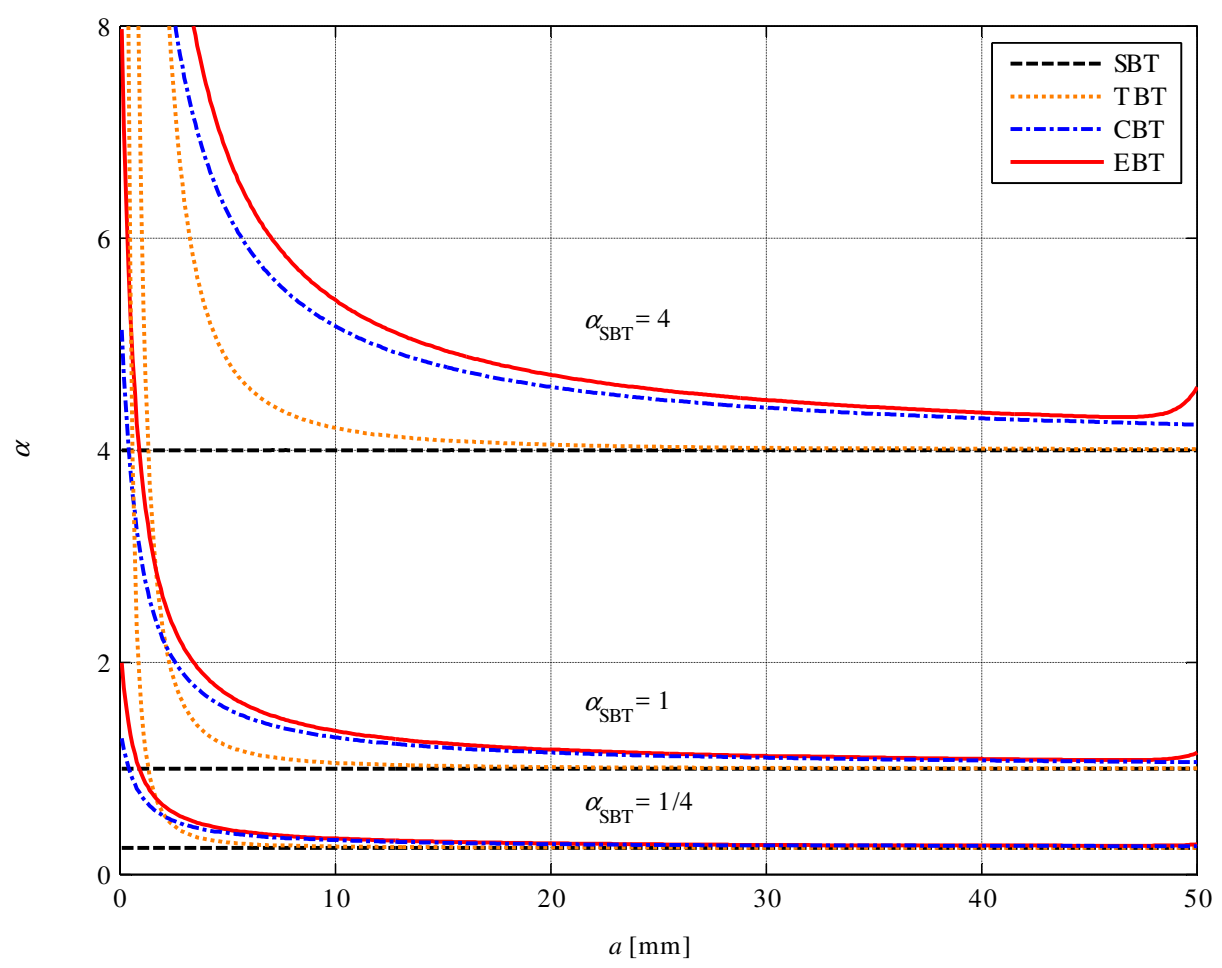

Fig. 11 Mixed-mode ratio vs. delamination length

\section{Application to experimental data reduction}

The concepts illustrated in the foregoing enable application of the EBT model of the MMB test to experimental data reduction, which essentially means extracting information about interlaminar fracture toughness from experimentally measured quantities. As a first step, the specimen's geometrical and mechanical properties should be determined as in the standard test procedure [5].

Next, it is necessary to estimate the values of the interface elastic constants, which can be accomplished by applying the compliance calibration strategy 
described in Section 3.5 with reference to the experimental results, instead of numerical simulations. To this aim, preliminary DCB and ENF tests should be carried out on the type of specimen in question. Then, the experimentally measured compliances, $C_{\mathrm{DCB}}^{\mathrm{EXP}}$ and $C_{\mathrm{ENF}}^{\mathrm{EXP}}$, should be entered into Eqs. (34), which are then numerically solved to obtain $k_{x}$ and $k_{z}$.

From this point on, the MMB test is carried out as usual. The load values related to crack initiation are recorded and used in Eqs. (49) to yield the critical mode I and II contributions to the energy release rate, $G_{\mathrm{Ic}}$ and $G_{\mathrm{IIc}}$, according to the EBT model. Equivalently, the crack length correction parameters can be computed from Eqs. (52) and used in the CBT relationships Eqs. (43).

By way of illustration, we consider an experimental study by Ducept et al. [32], who report on the results of MMB tests on glass/epoxy unidirectional laminated specimens. The considered specimen has span $L=130 \mathrm{~mm}$, width $B=$ $20 \mathrm{~mm}$, and thickness $H=2 h=5 \mathrm{~mm}$; the initial delamination length is $a=35 \mathrm{~mm}$. The elastic moduli of the material are $E_{x}=25.7 \mathrm{GPa}, E_{y}=6.5 \mathrm{GPa}$, and $G_{x y}=2.5 \mathrm{GPa}$. Preliminary DCB and ENF tests were performed, from which the compliances $\quad C_{\mathrm{DCB}}^{\mathrm{EXP}}=2.8 \mathrm{~mm} / 49 \mathrm{~N}=0.057 \mathrm{~mm} / \mathrm{N} \quad$ and $C_{\mathrm{ENF}}^{\mathrm{EXP}}=6.5 \mathrm{~mm} / 600 \mathrm{~N}=0.011 \mathrm{~mm} / \mathrm{N}$ were determined. Calibration with the corresponding predictions of the EBT model yields the values of the interface constants $k_{x}=17500 \mathrm{~N} / \mathrm{mm}^{3}$ and $k_{z}=1675 \mathrm{~N} / \mathrm{mm}^{3}$.

Table 2 Interlaminar fracture toughness values $\left[\mathrm{J} / \mathrm{m}^{2}\right]$ deduced from DCB, MMB, and ENF tests for unidirectional laminated specimens [32]

\begin{tabular}{|c|c|c|c|c|c|c|c|c|c|c|}
\hline & DCB & \multicolumn{2}{|c|}{ MMB } & \multicolumn{2}{|c|}{ MMB } & \multicolumn{2}{|c|}{ MMB } & \multicolumn{2}{|c|}{ MMB } & ENF \\
\hline$\alpha_{\mathrm{SBT}}$ & $\infty$ & \multicolumn{2}{|c|}{3} & \multicolumn{2}{|c|}{1} & \multicolumn{2}{|c|}{$1 / 3$} & \multicolumn{2}{|c|}{$1 / 8$} & 0 \\
\hline$c[\mathrm{~mm}]$ & - & \multicolumn{2}{|c|}{108.3} & \multicolumn{2}{|c|}{56.8} & \multicolumn{2}{|c|}{39} & \multicolumn{2}{|c|}{31.5} & - \\
\hline \multirow[t]{2}{*}{$P_{\mathrm{c}}[\mathrm{N}]$} & 49 & \multicolumn{2}{|c|}{60} & \multicolumn{2}{|c|}{145} & \multicolumn{2}{|c|}{256} & \multicolumn{2}{|c|}{355} & 600 \\
\hline & $G_{\mathrm{Ic}}$ & $G_{\mathrm{Ic}}$ & $G_{\text {IIc }}$ & $G_{\mathrm{Ic}}$ & $G_{\mathrm{IIc}}$ & $G_{\mathrm{Ic}}$ & $G_{\text {IIc }}$ & $G_{\mathrm{Ic}}$ & $G_{\text {IIc }}$ & $G_{\text {IIc }}$ \\
\hline SBT & 220 & 329 & 110 & 317 & 317 & 240 & 720 & 149 & 1192 & 1510 \\
\hline TBT & 221 & 331 & 110 & 319 & 317 & 241 & 720 & 150 & 1192 & 1510 \\
\hline CBT & 259 & 388 & 118 & 373 & 340 & 283 & 772 & 175 & 1279 & 1620 \\
\hline EBT & 267 & 400 & 114 & 385 & 329 & 291 & 748 & 181 & 1239 & 1569 \\
\hline
\end{tabular}




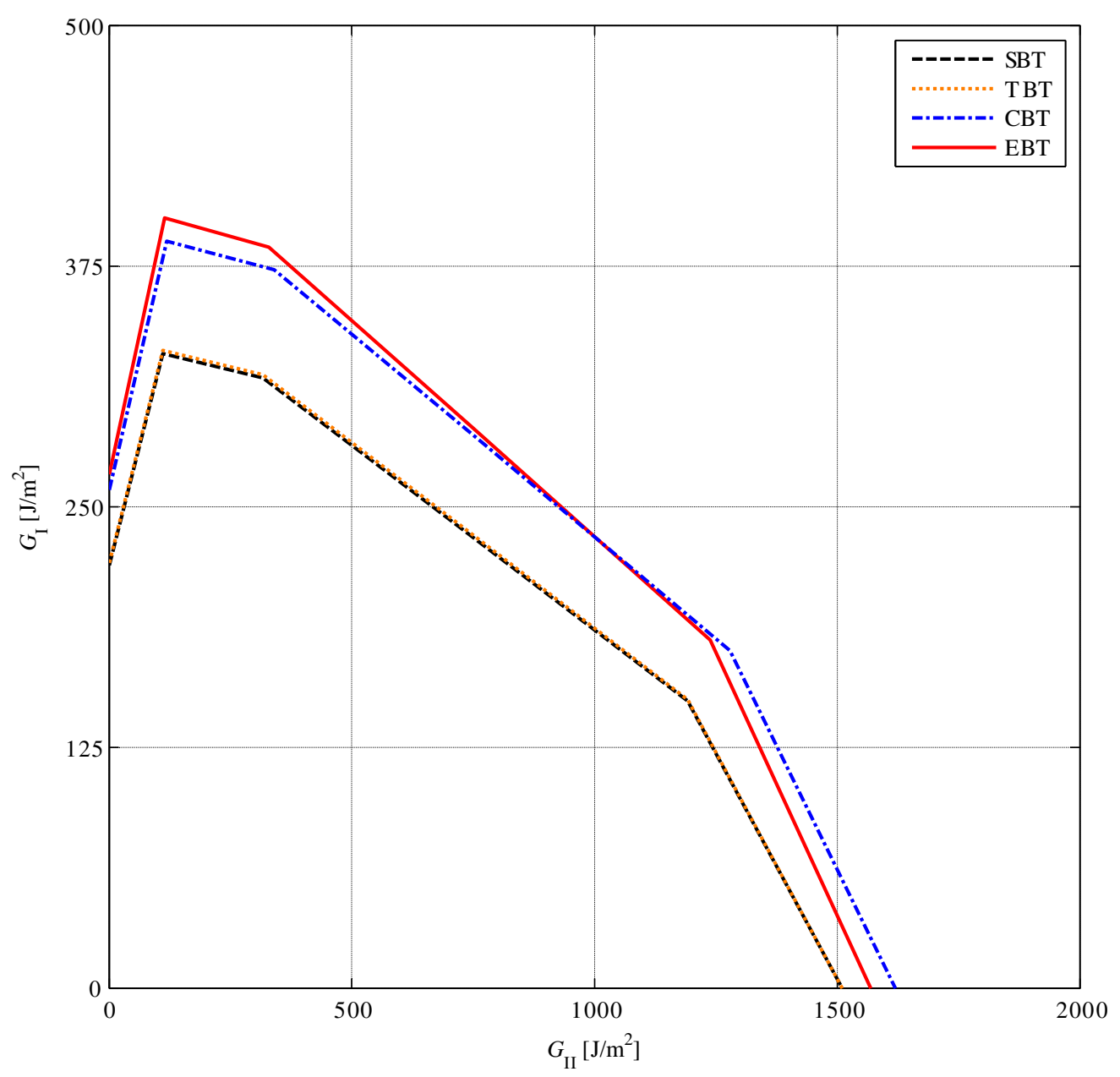

Fig. 12 Mixed-mode interlaminar fracture initiation envelopes

Table 2 shows the loads related to crack initiation (deduced using the values and expressions reported in the original study [32]), together with the values of $G_{\text {Ic }}$ and $G_{\text {IIc }}$ as predicted by the SBT, TBT, CBT, and EBT models. Figure 12 represents the corresponding mixed-mode interlaminar fracture initiation envelopes in the plane of $G_{\mathrm{I}}$ and $G_{\mathrm{II}}$. The SBT and TBT models yield nearly coincident envelopes. Instead, the envelopes of the CBT and EBT models - both markedly less conservative than the simpler models - are comparable with each other, except for some minor discrepancies.

As a first example application to multidirectional laminated specimens, we consider the numerical and experimental results presented by Pereira and de Morais for DCB, ENF, and MMB tests on glass/epoxy [17] and carbon/epoxy [19] composites. All the considered specimens have stacking sequence $\left[\left(0_{2} / 90\right)_{6} / 0_{2} / /\left(0_{2} / 90\right)_{6} / 0_{2}\right]$, where // denotes the position of the delamination. The elastic moduli of each lamina are listed in Table 3 . The extensional stiffness, shear 
stiffness and bending stiffness of the laminates have been computed according to classical laminated plate theory [21] and are given in Table 4. All specimens have width $B=20 \mathrm{~mm}$ and thickness $H=2 h=6 \mathrm{~mm}$, while the remaining geometric properties are given in Table 5. The finite element analyses performed by Pereira [33] for the glass/epoxy specimens furnished $C_{\mathrm{DCB}}^{\mathrm{FEM}}=0.551939 \mathrm{~mm} / \mathrm{N}$ and $C_{\mathrm{ENF}}^{\mathrm{FEM}}=0.002492 \mathrm{~mm} / \mathrm{N}$, whence $k_{x}=6146.8 \mathrm{~N} / \mathrm{mm}^{3}$ and $k_{z}=4578.0 \mathrm{~N} / \mathrm{mm}^{3}$ were determined. Likewise, for the carbon/epoxy specimens the values of compliance $C_{\mathrm{DCB}}^{\mathrm{FEM}}=0.063001 \mathrm{~mm} / \mathrm{N} \quad$ and $\quad C_{\mathrm{ENF}}^{\mathrm{FEM}}=0.000809 \mathrm{~mm} / \mathrm{N} \quad$ yielded $k_{x}=12735.5 \mathrm{~N} / \mathrm{mm}^{3}$ and $k_{z}=7764.8 \mathrm{~N} / \mathrm{mm}^{3}$. Table 6 reports the crack length correction parameters computed according to the CBT model, using Eqs. (20) with the homogenised flexural and shear moduli [18], and according to the EBT model, using Eqs. (52).

Table 3 Elastic moduli of a single lamina [33]

\begin{tabular}{cccc}
\hline Material & $\begin{array}{c}E_{1} \\
{[\mathrm{GPa}]}\end{array}$ & $\begin{array}{c}E_{2} \\
{[\mathrm{GPa}]}\end{array}$ & $\begin{array}{c}G_{12} \\
{[\mathrm{GPa}]}\end{array}$ \\
\hline glass / epoxy & 33 & 19 & 4.8 \\
\hline carbon / epoxy & 130 & 8.2 & 4.1 \\
\hline
\end{tabular}

Table 4 Laminates stiffnesses for multidirectional laminated specimens

\begin{tabular}{cccc}
\hline Material & $\begin{array}{c}A_{1} \\
{[\mathrm{~N} / \mathrm{mm}]}\end{array}$ & $\begin{array}{c}C_{1} \\
{[\mathrm{~N} / \mathrm{mm}]}\end{array}$ & $\begin{array}{c}D_{1} \\
{[\mathrm{~N} \mathrm{~mm}]}\end{array}$ \\
\hline glass / epoxy & 86400.0 & 10169.5 & 66784.5 \\
\hline carbon / epoxy & 280380.0 & 9129.9 & 227550.2 \\
\hline
\end{tabular}

Table 5 Geometric properties of multidirectional laminated specimens [33]

\begin{tabular}{cccc}
\hline Material & Test & $\begin{array}{c}\ell \\
{[\mathrm{mm}]}\end{array}$ & $\begin{array}{c}a \\
{[\mathrm{~mm}]}\end{array}$ \\
\hline \multirow{2}{*}{ glass / epoxy } & DCB & 135 & 100 \\
\cline { 2 - 4 } & ENF & 50 & 25 \\
\hline carbon / epoxy & DCB & 135 & 70 \\
\cline { 2 - 4 } & ENF & 50 & 25 \\
\hline
\end{tabular}


Table 6 Crack-length correction parameters for multidirectional laminated specimens

\begin{tabular}{cccc}
\hline Material & Model & $\chi_{\mathrm{I}}$ & $\chi_{\text {II }}$ \\
\hline \multirow{2}{*}{ glass / epoxy } & CBT & 1.051 & 0.441 \\
\cline { 2 - 4 } & EBT & 1.153 & 0.447 \\
\hline carbon / epoxy & CBT & 1.857 & 0.780 \\
\cline { 2 - 4 } & EBT & 1.903 & 0.569 \\
\hline
\end{tabular}

\section{Conclusions}

The analytical solution to the enhanced beam-theory model of the MMB test formulated in Part I of this paper [7] has been applied to deduce analytical expressions for the specimen's compliance, energy release rate, and mode mixity.

The specimen's compliance, $C$, has been defined, and alternative expressions for it have been deduced and discussed. Particular attention has been devoted to illustrating how the displacements of DCB and ENF tests should be superimposed to correctly determine the displacements of the MMB test. The compliance predictions of the EBT model have been compared with those of the SBT, TBT, and CBT models, as well as with the results of finite element analyses. Only the EBT model has proved capable of furnishing a close match with the finite element results by taking into account crack-tip deflections and rotations (thanks to the deformable interface), as well as shear deformation of the sublaminates.

The energy release rate, $G$, has also been defined and analytical expressions for it deduced. The presence of an elastic-brittle interface connecting the sublaminates in the EBT model has allowed for straightforward evaluation of the modal contributions, $G_{\mathrm{I}}$ and $G_{\mathrm{II}}$, based on the peak values of the interfacial stresses at the crack tip. The energy release rate predictions of the EBT model have been compared with those of the SBT, TBT, and CBT models, and turn out to be in very good agreement with the last. The CBT model, however, is based on crack length correction parameters, which are strictly defined only for homogenous orthotropic specimens. Instead, the EBT model can be applied to laminated specimens having generic stacking sequences. Thus, it has been possible to deduce analytical expressions for the crack length correction parameters that hold for both unidirectional and multidirectional laminated specimens. 
Lastly, the mode mixity characterising the relative amount of fracture modes I and II during crack propagation has been defined, and expressions have been deduced for the mode-mixity angle, $\psi$, and mixed-mode ratio, $\alpha$. The conditions necessary for pure fracture modes have also been discussed. The mode mixity predictions of the EBT model have been compared with those of the SBT, TBT, and CBT models, and turn out to be in good agreement with the last.

The enhanced beam-theory model has proved capable of furnishing accurate predictions for the main quantities involved in the interpretation of MMB test results. The predictive effectiveness of the model, however, rests crucially on reliable estimation of the interface elastic constants. In the paper, we have explained first how the interface constants can be evaluated through a compliance calibration strategy implemented through a finite element model and then shown how the same strategy can be employed to determine the interface parameters from experimental results.

\section{Acknowledgements}

The financial support of the Italian Ministry of Education, University and Research (MIUR) under programme PRIN 2008 "Light structures based on multiscale material in civil engineering: stiffness and strength, assembly and industrial repeatability" (Prot. N. 20089RJKYN_002) is gratefully acknowledged.

\section{References}

1. Crews JH Jr, Reeder JR (1988) A mixed-mode bending apparatus for delamination testing. NASA TM-100662.

URL http://ntrs.nasa.gov/archive/nasa/casi.ntrs.nasa.gov/19890001574_1989001574.pdf

2. Reeder JR, Crews JH Jr (1990) Mixed-mode bending method for delamination testing. AIAA J 28(7):1270-1276. doi: http://dx.doi.org/10.2514/3.25204

3. Reeder JR, Crews JH Jr (1992) Redesign of mixed-mode bending delamination test to reduce nonlinear effects. J Compos Tech Res 14(1):12-19. doi: http://dx.doi.org/10.1520/CTR10078J

4. Reeder JR (2003) Refinements to the mixed-mode bending test for delamination toughness. J Compos Tech Res 25(4):191-195. doi: http://dx.doi.org/10.1520/CTR10961J

5. ASTM (2006) Standard Test Method for Mixed Mode I-Mode II Interlaminar Fracture Toughness of Unidirectional Fiber Reinforced Polymer Matrix Composites, D6671/D6671M06. American Society for Testing and Materials, West Conshohocken, PA.

doi: http://dx.doi.org/10.1520/D6671_D6671M-06 
6. Adams DF, Carlsson LA, Pipes RB (2003) Experimental Characterization of Advanced Composite Materials $-3^{\text {rd }}$ edition. CRC Press, Boca Raton

7. Bennati S, Fisicaro P, Valvo PS (2013) An enhanced beam-theory model of the mixed-mode bending (MMB) test - Part I: literature review and mechanical model. Meccanica 48(2):443462. doi: http://dx.doi.org/10.1007/s11012-012-9686-3

8. Kinloch AJ, Wang Y, Williams JG, Yayla P (1993) The mixed-mode delamination of fibre composite materials. Compos Sci Technol 47(3):225-237. doi: http://dx.doi.org/10.1016/0266-3538(93)90031-B

9. Williams JG (1989) End corrections for orthotropic DCB specimens. Compos Sci Technol 35(4):367-376. doi: http://dx.doi.org/10.1016/0266-3538(89)90058-4

10. Kanninen MF (1973) An augmented double cantilever beam model for studying crack propagation and arrest. Int J Fract 9(1):83-92. doi: http://dx.doi.org/10.1007/BF00035958

11. Wang Y, Williams JG (1992) Corrections for mode II fracture toughness specimens of composites materials. Compos Sci Technol 43(3):251-256.

doi: http://dx.doi.org/10.1016/0266-3538(92)90096-L

12. Bhashyan S, Davidson BD (1997) Evaluation of data reduction methods for the mixed mode bending test. AIAA J 35(3):546-552. doi: http://dx.doi.org/10.2514/2.129

13. Wang JL, Qiao PZ (2004) Novel beam analysis of end notched flexure specimen for mode-II fracture. Engrg Fract Mech 71(2):219-231.

doi: http://dx.doi.org/10.1016/S0013-7944(03)00096-1

14. de Morais AB (2011) Novel cohesive beam model for the End-Notched Flexure (ENF) specimen. Engrg Fract Mech 78(17):3017-3029.

doi: http://dx.doi.org/10.1016/j.engfracmech.2011.08.019

15. Jumel J, Budzik MK, Ben Salem N, Shanahan MER (2013) Instrumented End Notched Flexure - Crack propagation and process zone monitoring. Part I: Modelling and analysis. Int J Solids Struct. 50(2):297-309. doi: http://dx.doi.org/10.1016/j.ijsolstr.2012.08.028

16. de Morais AB, Pereira AB (2006) Mixed mode I + II interlaminar fracture of glass/epoxy multidirectional laminates - Part 1: Analysis. Compos Sci Technol 66(13):1889-1895. doi: http://dx.doi.org/10.1016/j.compscitech.2006.04.006

17. Pereira AB, de Morais AB (2006) Mixed mode I + II interlaminar fracture of glass/epoxy multidirectional laminates - Part 2: Experiments. Compos Sci Technol 66(13):1896-1902. doi: $\underline{\text { http://dx.doi.org/10.1016/j.compscitech.2006.04.008 }}$

18. de Morais AB, Pereira AB (2007) Interlaminar fracture of multidirectional glass/epoxy laminates under mixed-mode I + II loading. Mech Compos Mater 43(3):233-244. doi: http://dx.doi.org/10.1007/s11029-007-0023-1

19. Pereira AB, de Morais AB (2008) Mixed mode I + II interlaminar fracture of carbon/epoxy laminates. Composites Part A 39(2):322-333.

doi: http://dx.doi.org/10.1016/j.compositesa.2007.10.013

20. Bennati S, Colleluori M, Corigliano D, Valvo PS (2009) An enhanced beam-theory model of the asymmetric double cantilever beam (ADCB) test for composite laminates. Compos Sci Technol 69(11-12): 1735-1745. doi: http://dx.doi.org/10.1016/j.compscitech.2009.01.019 
21. Jones RM (1999) Mechanics of composite materials $-2^{\text {nd }}$ edition. Taylor \& Francis Inc., Philadelphia, PA

22. Vannucci P, Verchery G (2001) A special class of uncoupled and quasi-homogeneous laminates. Compos Sci Technol 61(10):1465-1473. doi: http://dx.doi.org/10.1016/S0266-3538(01)00039-2

23. Ozdil F, Carlsson LA (1999) Beam analysis of angle-ply laminate mixed-mode bending specimens. Compos Sci Technol 59(6):937-945. doi: http://dx.doi.org/10.1016/S0266-3538(98)00128-6

24. Timoshenko SP (1984) Strength of materials: Elementary Theory and Problems - Vol. 1. Krieger Publishing, Melbourne, FL

25. Strand7 (2005) Theoretical manual: theoretical background to the Strand7 finite element analysis system $-1^{\text {st }}$ edn G+D Computing, Sidney

26. Hutchinson JW, Suo Z (1991) Mixed mode cracking in layered materials. Adv Appl Mech 29(C):63-191. doi: http://dx.doi.org/10.1016/S0065-2156(08)70164-9

27. Carlsson LA, Gillespie JW, Pipes RB (1986) On the analysis and design of the end notched flexure (ENF) specimen for Mode II testing. J Compos Mater 20(6):594-604.

doi: http://dx.doi.org/10.1177/002199838602000606

28. Fan C, Ben Jar P-Y, Cheng J-JR (2006) Revisit the analysis of end-notched-flexure (ENF) specimen. Compos Sci Technol 66(10):1497-1498.

doi: http://dx.doi.org/10.1016/j.compscitech.2006.01.016

29. Valvo PS (2008) Does shear deformability influence the mode II delamination of laminated beams?. In: ECF $17-17^{\text {th }}$ European Conference on Fracture. 2-5 September 2008, Brno, Czech Republic

30. Corigliano A (1993) Formulation, identification and use of interface models in the numerical analysis of composite delamination. Int J Solids Struct 30(20):2779-2811. doi: http://dx.doi.org/10.1016/0020-7683(93)90154-Y

31. Budzik MK, Jumel J, Ben Salem N, Shanahan MER (2013) Instrumented End Notched Flexure - Crack propagation and process zone monitoring. Part II: Data reduction and experimental. Int J Solids Struct. 50(2):310-319. doi: http://dx.doi.org/10.1016/j.ijsolstr.2012.08.030

32. Ducept F, Davies P, Gamby D (1997) An experimental study to validate tests used to determine mixed mode failure criteria of glass/epoxy composites. Compos Part A 28(8):719729. doi: http://dx.doi.org/10.1016/S1359-835X(97)00012-2

33. Pereira AB (2006) Fractura interlaminar de compósitos de matriz polimérica. Ph.D Thesis, University of Aveiro. URL http://ria.ua.pt/bitstream/10773/2549/1/2007001048.pdf 